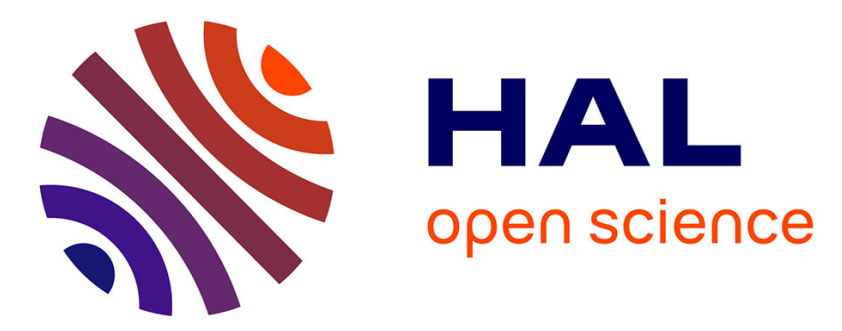

\title{
Short synthesis of new salacinol analogues and their evaluation as glycosidase inhibitors
}

Estelle Gallienne, M. Benazza, G. Demailly, Jean Bolte, Marielle Lemaire

\section{To cite this version:}

Estelle Gallienne, M. Benazza, G. Demailly, Jean Bolte, Marielle Lemaire. Short synthesis of new salacinol analogues and their evaluation as glycosidase inhibitors. Tetrahedron, 2005, pp.4557-4568. 10.1016/j.tet.2005.03.015 . hal-00125713

\section{HAL Id: hal-00125713 \\ https://hal.science/hal-00125713}

Submitted on 2 Mar 2007

HAL is a multi-disciplinary open access archive for the deposit and dissemination of scientific research documents, whether they are published or not. The documents may come from teaching and research institutions in France or abroad, or from public or private research centers.
L'archive ouverte pluridisciplinaire HAL, est destinée au dépôt et à la diffusion de documents scientifiques de niveau recherche, publiés ou non, émanant des établissements d'enseignement et de recherche français ou étrangers, des laboratoires publics ou privés. 


\title{
Short synthesis of new salacinol analogues and their evaluation as glycosidase inhibitors
}

\author{
Estelle Gallienne, ${ }^{\mathrm{a}}$ Mohammed Benazza, ${ }^{\mathrm{b},}$ Gilles Demailly, ${ }^{\mathrm{b}}$ Jean Bolte $^{\mathrm{a}}$ \\ and Marielle Lemaire ${ }^{\mathrm{a}, *}$ \\ ${ }^{a}$ Laboratoire SEESIB, UMR 6504,Université Blaise Pascal, 24 av des Landais, 63177 Aubière cedex, France \\ ${ }^{\mathrm{b}}$ Laboratoire des Glucides, FRE 2779, Ilot des Poulies, Université de Picardie Jules Verne, 33 rue Saint-Leu, 80039 Amiens cedex, France
}

Received 16 December 2004; revised 1 March 2005; accepted 3 March 2005

Available online 23 March 2005

Abstract-Versatile synthesis of some analogues of the naturally-occurring $\alpha$-glucosidase inhibitor salacinol (1), involving thioanhydro alditol moieties with erythro, D, L-threo, xylo, ribo, D-arabino and D-manno configurations is described. Nucleophilic attack at the leasthindered carbon atom of an L- or D-protected erythritol cyclic sulfate by the thioanhydro alditol sulfur atom yielded the desired zwitterionic compounds. In addition, the preparation of the cyclic sulfates of 2,4- $O$-benzylidene-D-erythritol and 2,4- $O$-isopropylidene-L-erythritol was improved. Enzyme inhibition tests showed that most of the new compounds were weak but specific inhibitors, while good inhibitory activity was found for a six-membered ring analogue $\left(\beta\right.$-glucosidase: $\left.K_{\mathrm{i}}=16 \mu \mathrm{M}\right)$.

\section{Introduction}

Salacinol 1 and kotalanol $\mathbf{2}$ are $\alpha$-glucosidase inhibitors isolated from the Hippocrateaceae plant Salacia reticulata WIGHT, a large woody climbing plant widespread in Sri Lanka and South India (Fig. 1). Extracts of this plant have been traditionally used in the Ayurvedic system of Indian medicine as a treatment for non-insulin-dependent diabetes. ${ }^{1}$ The methanol extract from the roots and stems of $S$. reticulata is reported to show inhibitory activity against the increase in serum glucose levels after the administration of sucrose or maltose in rats. ${ }^{1 b}$ It was demonstrated that $\mathbf{1}$ and $\mathbf{2}$ were responsible for this

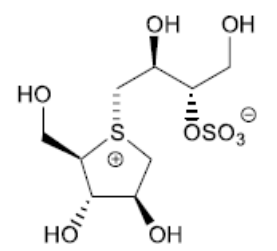

salacinol 1

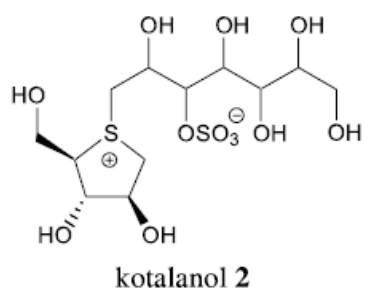

Figure 1.
Keywords: Salacinol; Glycosidsase inhibitors; Cyclic sulfate; Thiaanhydroalditol; Zwitterion; Sulfonium sulfate inner salt.

* Corresponding author. Tel.: + 334734075 84; fax: + 334734077 17; e-mail: marielle.lemaire@chimie.univ-bpclermont.fr

${ }^{\dagger}$ Tel.: +33 322827527 ; fax: + 33322827560 . inhibitory activity. ${ }^{\text {la,c }}$ These novel glycosidase inhibitors have unique zwitterionic structures in which the sulfonium cation is stabilized by the sulfate anion. It is assumed that the sulfonium center permanently mimics the incremental positive charge that forms at both the ring oxygen and the anomeric carbon of the glycoside during hydrolysis in the active site of a glycosidase. Variation of the chiral centers and/or ring sizes would be expected to modulate the binding interactions and consequently modulate the specificity towards the glycosidases. ${ }^{2,3}$ In view of both its very high glycosidase inhibitory activity and its novel structure, chemists have conducted much research on the total synthesis of $\mathbf{1}$ and its analogues. As the absolute configurations of the kotalanol $\mathbf{2}$ side chain have not yet been established, all the work has focused on salacinol. ${ }^{3-7}$ Nitrogen and selenium analogues have also been described. . $^{3,6,8-10}$

All the strategies described in the literature to obtain the zwitterionic moiety are based on the same reaction: the nucleophilic attack of the heteroatom of a protected or unprotected polyhydroxylated heterocycle at the leasthindered carbon atom of an L- or D-protected erythritol cyclic sulfate (Scheme 1). L-protected erythritol provides the side chain of salacinol and D-protected erythritol its enantiomer.

In 2000, Yuasa et al. were the first to present the synthesis of salacinol 1 and its diastereoisomer 3 (Fig. 2). ${ }^{4}$ Later, 


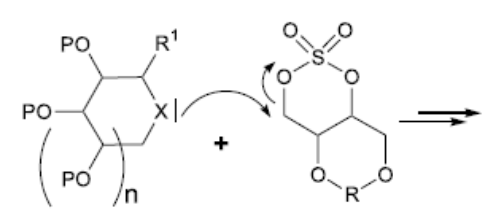

$\mathrm{n}=0,1,2 ; \mathrm{R}=\mathrm{CH}-\mathrm{Ph}, \mathrm{C}\left(\mathrm{CH}_{3}\right)_{2}$

$\mathrm{R}^{1}=\mathrm{H}, \mathrm{CH}_{2} \mathrm{OP} \quad \mathrm{X}=\mathrm{S}, \mathrm{NH}, \mathrm{Se} ; \mathrm{P}=$ protecting group or $\mathrm{H}$

Scheme 1. General strategy.

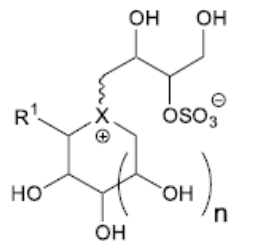

Preparations of the corresponding cyclic sulfite with good yields have been described $\left(82 \%,{ }^{5}>95 \%{ }^{9}\right)$, but we failed to reproduce these results even after numerous attempts. Our yields were around 55 to $60 \%$; two syntheses of such cyclic sulfite/sulfate are reported with $60 \%$ yield $^{13}$ and $62 \%$ yield. $^{4}$

isolated. To shorten this part of the synthesis, we performed the periodidate oxidation and the reduction with $\mathrm{NaBH}_{4}$ in one pot. Iodine was also formed but could easily be reduced by treatment with sodium thiosulfate. The diol $\mathbf{9}$ was thereby isolated in very high yield $(92 \%)$.
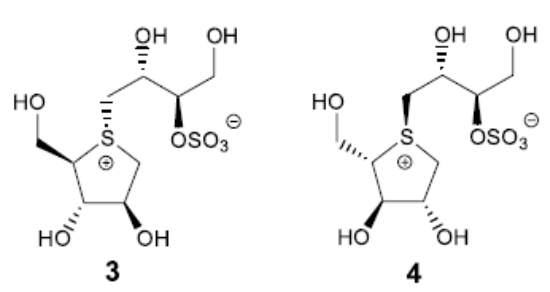

Figure 2. Salacinol stereoisomers and six-membered ring analogues.

Ghavami et al. reported their own synthesis of $\mathbf{1}$, its enantiomer $\mathbf{4}$ and the diastereoisomers $\mathbf{3}$ and $\mathbf{5}$ (Fig. 2). ${ }^{5-7}$ More recently the same group extended this method to prepare other sulfonium analogues with two different sixmembered rings, ${ }^{3}$ one of them obtained from alditols using the procedure described by Benazza et al. ${ }^{11}$ Never observed with the five-membered ring, the coupling reaction with a six-membered ring resulted in the formation of two configurations at the sulfur atom. In the series with no hydroxymethyl group, the epimers were separated and four diastereoisomers were obtained (6 is given as an example, Fig. 2). When the hydroxymethyl group was present, the separation was not possible and mixtures of epimers (e.g., 7) were obtained (Fig. 2). ${ }^{3}$

To add to this new class of glycosidase inhibitors, we undertook the synthesis of several salacinol analogues obtained with the same general strategy (Scheme 1). For further structure-function studies, we modulated the ring size and its stereocenters, and kept the same L-erythritol sulfated side chain as salacinol and its enantiomer derived from the D-erythritol. Inhibition activities towards six commercial glycosidases are presented for all the compounds synthesized.

\section{Results and discussion}

We synthesized the protected D- and L-erythritol cyclic sulfates from 4,6- $O$-benzylidene-D-glucose ${ }^{12}$ by a modified procedure previously reported with no experimental section or description of compounds by Muraoka et al. (Scheme 2). ${ }^{9}$ The L stereoisomer synthesis has also been described from L-glucose as starting material. ${ }^{4,5}$ To obtain large quantities of both 10 and $\mathbf{1 3}$, the following reactions were performed at a scale of several grams.

In the standard protocol, the aldehyde obtained from $\mathrm{NaIO}_{4}$ oxidation of the 4,6-O-benzylidene-D-glucose is usually
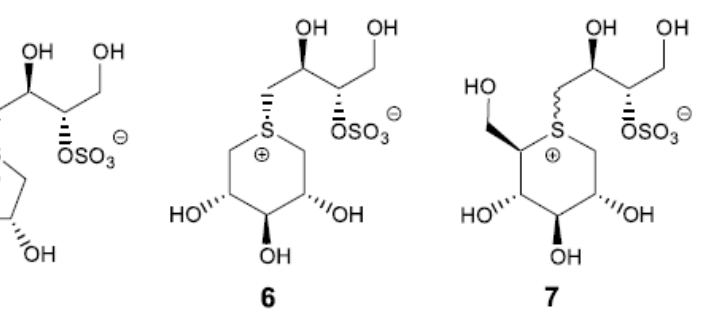

We observed that this cyclic sulfite was partially unstable in workup conditions and purification steps. Hydrolysis of the sulfite occurred easily and starting diol was isolated, although it was not present at the end of the reaction (from TLC). To prevent this side reaction, and as the reaction with $\mathrm{SOCl}_{2}$ was total, we oxidized the sulfite to the sulfate $\mathbf{1 0}$ after a simple evaporation of the crude reaction mixture under vacuum. The non-isolated sulfite was converted into sulfate $\mathbf{1 0}$ with $80 \%$ overall yield.

The diol 9 also afforded the L-erythritol series when correctly protected by acetonide and then subjected to hydrogenolysis over Pd/C. The diol 12 was isolated in $84 \%$ yield from 9.

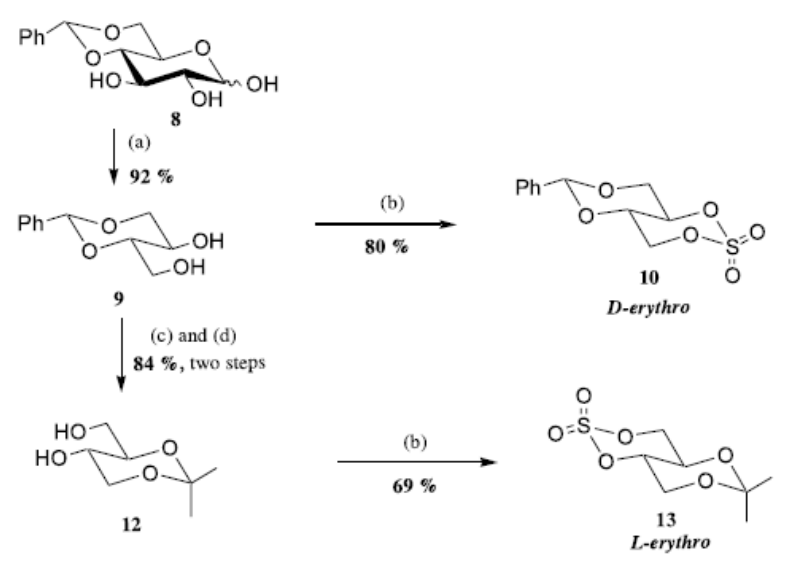

Scheme 2. Reagents and conditions: (a) $\mathrm{NaIO}_{4}, \mathrm{NaHCO}_{3}, \mathrm{H}_{2} \mathrm{O}$, rt then $\mathrm{NaBH}_{4}, \mathrm{H}_{2} \mathrm{O} / \mathrm{EtOH}$, rt; (b) $\mathrm{SOCl}_{2}$, anh. $\mathrm{NEt}_{3}, \mathrm{CH}_{2} \mathrm{Cl}_{2}, 0^{\circ} \mathrm{C}$ then $\mathrm{RuCl}_{3}$, $\mathrm{NaIO}_{4}, \mathrm{CH}_{2} \mathrm{Cl}_{2} / \mathrm{CH}_{3} \mathrm{CN} / \mathrm{H}_{2} \mathrm{O}$, rt; (c) $\mathrm{CH}_{3}\left(\mathrm{OCH}_{3}\right) \mathrm{C}=\mathrm{CH}_{2}$, TsOH, DMF, $0{ }^{\circ} \mathrm{C}$; (d) 11, $\mathrm{H}_{2}, \mathrm{Pd} / \mathrm{C}, \mathrm{EtOH}, \mathrm{rt}$.

Instability of the corresponding cyclic sulfite was also observed, and was even more marked. This problem was also solved by applying our one-pot method. The crude cyclic sulfite obtained after concentration under vacuum 


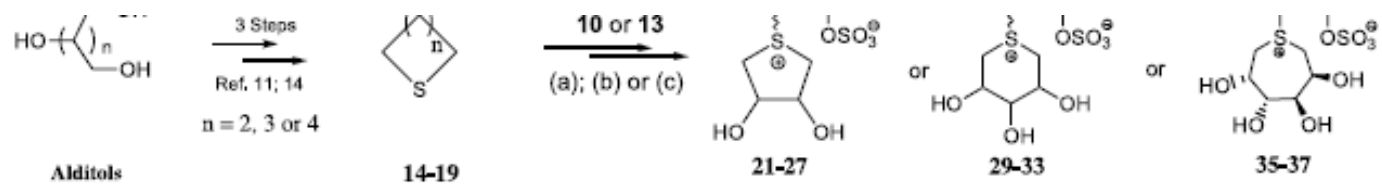

Scheme 3. Reagents and conditions: (a) HFIP, $\mathrm{Na}_{2} \mathrm{CO}_{3}$, reflux; (b) $\mathrm{H}_{2} \mathrm{O}, \mathrm{H}^{+}$(Dowex $50 \mathrm{~W} \times 8$ ), $\mathrm{rt}$; (c) $\mathrm{H}_{2}, \mathrm{Pd} / \mathrm{C}, \mathrm{AcOH} / \mathrm{H}_{2} \mathrm{O}, \mathrm{rt}$.

was oxidized to give 13 in $69 \%$ yield. With these three onepot operations, we shortened the total synthesis of the desired sulfates and notably increased the yields for their preparation.

The polyhydroxylated thiaheterocycles 14 to 19 (Table 1 , Scheme 3) used in these experiments were prepared by Benazza et al. They were obtained from expeditious reactions of acetylated $\alpha, \omega$-dibromoalditols with sodium sulfide. ${ }^{11,14}$ With tetritols as substrates the bis-cyclic sulfates ${ }^{15 a}$ or the more recently described bis-cyclic thionocarbonates $^{15 \mathrm{~b}}$ were also used as bis-electrophilic intermediates.

The coupling reactions (Scheme 3) were all performed in hexafluoroisopropanol (HFIP) as Ghavami et al. had demonstrated its efficiency in such reactions. ${ }^{7}$ Addition of sodium carbonate increases the stability of the sulfate under reflux. ${ }^{5,16}$ The results are presented in Table 1 . In some cases (entries $1,2,6,8,10$ ), an $R / S$ stereocenter was created on the sulfur atom and our mixtures were not separable. The compounds are all characterized as mixtures of the two configurations. In other cases (entries 3, 4, 11 and 12), the sulfur atom is not stereogenic, correlated to the $\mathrm{C} 2$ axis of the starting thiaheterocycles. As compound $\mathbf{1 5}$ is racemic, two diastereoisomers were obtained after coupling reactions corresponding to the structures $24 \mathrm{a}$ and $24 \mathrm{~b}$, and $26 \mathrm{a}$ and 26b (entries 3 and 4). These mixtures were also not separable. As compound 19 is optically pure, only one diastereoisomer was isolated with $\mathbf{1 0}$ (compound 36) and with 13 (compound 34) (entries 11 and 12).

Based on the wide differences in yields obtained, the thiaheterocycle reactivities seemed to depend on the ring size and configurations of asymmetric carbon atoms. Thus with the five-membered ring (entries 1 to 4 ) the yields for zwitterionic compounds were good to excellent (from 62 to $95 \%$ ). In contrast, the three six-membered rings tested reacted poorly (entries 5 to 10). The coupling reaction did not take place with the L-erythritol cyclic sulfate $\mathbf{1 3}$ (entries 5,7 and 9) or with the compound 10 (entries 6,8 and 10), the yields were modest (except with 17: 60\%, entry 7). The sulfur atom of the seven-membered ring 19 (entries 11 and 12) did not react as well as the five-membered one but the two cyclic sulfates could even so be coupled to give the desired zwitterionic molecules. As for the six-membered ring, the reaction was much more efficient with the benzylidene protected cyclic sulfate than with acetonide protected compound $\mathbf{1 3}$.

We suggest that this effect may be attributed to the size and shape of the thiaheterocycle ring, resulting in nucleophilicity variation of the sulfur atom. In addition, the steric hindrance was greater for the methyl in the equatorial position than for the phenyl. Thus the accessibility of the nucleophile at the least-hindered carbon atom of the cyclic sulfate was reduced.

The last step was the diol deprotection. Two methods were used: hydrolysis under acidic conditions, and benzylidene hydrogenolysis over $\mathrm{Pd} / \mathrm{C}$. All the reactions were long, several days at room temperature. For the acid hydrolysis, we found it more convenient to use a Dowex resin as this can be easily removed by filtration (entries 1, 3, 6, 8, 10-12). With the hydrogenolysis over $\mathrm{Pd} / \mathrm{C}$, after $48 \mathrm{~h}$ the reaction mixture had to be filtered to eliminate $\mathrm{Pd} / \mathrm{C}$ and fresh catalyst was added (entries 2 and 4). Probably, traces of sulfur compounds or other impurities had poisoned the catalyst. Given this difficulty and the longer reaction time, the benzylidene acidic cleavage was preferred (entries 6,10 and 12). All the yields obtained (from 23 to $82 \%$ ) are comparable to those in the literature ${ }^{3,5}$ and were not optimized. Except for compounds $2 \mathbf{2 9}^{3}$ all the compounds synthesized are new salacinol analogues and were fully characterized.

\section{Inhibition studies}

The new salacinol analogues and the thiosugars were screened against six commercial glycosidases. The results for the zwitterions are given in Table 2 and are compared with the reported activity of salacinol $1 .{ }^{17}$ This compound presents a specificity among the glycosidase inhibitors. It is very active against disaccharidases such as $\alpha$-glucosidase from rice and inactive against other glucosidases or mannosidase. All the thiosugars tested are inactive. The compounds 21 to 27 , which contain the five-membered ring, are inactive against the $\alpha$-glucosidase from rice. As salacinol is a good inhibitor of this enzyme $\left(\mathrm{IC}_{50}=1.1 \times\right.$ $10^{-3} \mathrm{mM}$ ), the hydroxymethyl group is essential to a good inhibition in this case. Except for compound 35, which was never active, all the compounds inhibited at least one enzyme. They were moderately selective but the activities found were weak, around 1 to $3 \mathrm{mM}$ except for compounds $29 \mathrm{a}$ and $29 \mathrm{~b}$, which exhibited a very high activity towards $\beta$-glucosidase. In the literature, these two compounds were reported to be inactive towards an $\alpha$-glucoamylase. ${ }^{3}$ All the compounds tested were inactive against $\alpha$-glucosidase from baker's yeast and $\alpha$-galactosidase from green coffee beans. Finally, we have shown that salacinol analogues can be not only $\alpha$-glucosidase inhibitors but can also be active toward $\beta$-glucosidases. 


\section{Conclusion}

We have prepared several new salacinol analogues in good overall yields using free heterocycles in coupling reactions. Furthermore, we have improved, in terms of step number and yields on a scale of several grams, the synthesis of the two key cyclic sulfate intermediates. The cyclic sulfates $\mathbf{1 0}$ and $\mathbf{1 3}$ were obtained efficiently and easily from inexpensive D-glucose in three and five steps, respectively. As the thiosugars are inactive against all the glycosidases tested, the zwitterionic structure is responsible for the detected inhibition. Following the results of inhibition activities shown in Table 2, the presence of the hydroxymethyl group in the D-arabinothiolane moiety of salacinol seems important for the effectiveness of the inhibition. The ring size also seems to play a role, as a six-membered ring was found to be very active towards $\beta$-glucosidase, compared with the five or seven-membered rings. This is probably due to both the presence of more than two hydroxyl groups and to the conformation change. Finally, in agreement with the reported literature results ${ }^{3,5,6}$ the alditol ring stereochemistry obviously plays an important part since among the three configurations tested for the six-membered ring analogues, only one was active.

\section{Experimental}

All the reactions were monitored by TLC with Merck 60F254 precoated silica $(0.2 \mathrm{~mm})$ on aluminium. Flash chromatography was performed with Merck Kieselgel 60 $(40-63 \mu \mathrm{m})$; the solvent systems are given $\mathrm{v} / \mathrm{v}$. $\mathrm{NEt}_{3}$ was distilled over $\mathrm{CaH}_{2}$. Melting points were measured with a Reichert microscope and are uncorrected. ${ }^{1} \mathrm{H}$ NMR $(400 \mathrm{MHz})$ and ${ }^{13} \mathrm{C}$ NMR $(100 \mathrm{MHz})$ spectra were recorded on a Bruker Avance 400 in $\mathrm{CDCl}_{3}, \mathrm{CD}_{3} \mathrm{OD}$ or $\mathrm{D}_{2} \mathrm{O}$ (see indication). Chemical shifts $(\delta)$ are reported in ppm and coupling constants are given in $\mathrm{Hz}$. IR spectra were recorded on a Perkin-Elmer FT-IR Paragon 500. Optical rotations were measured on a JASCO DIP-370 polarimeter with a sodium $(589 \mathrm{~nm})$ lamp at $25^{\circ} \mathrm{C}$. High resolution mass spectra (HRMS) were recorded by the Centre Régional de Mesures Physiques de l'Ouest, Rennes.

\subsection{Synthesis of cyclic sulfates 10 and 13}

5.1.1. 2,4-O-Benzylidene-D-erythritol 9. To a solution of 4,6 - $O$-benzylidene-D-glucose ${ }^{12} 8(10.0 \mathrm{~g}, 37.4 \mathrm{mmol})$ in $70 \mathrm{~mL}$ of water was added a solution of $\mathrm{NaIO}_{4}(16.1 \mathrm{~g}$, $75.4 \mathrm{mmol})$ and $\mathrm{NaHCO}_{3}(3.17 \mathrm{~g}, 37.7 \mathrm{mmol})$ in $130 \mathrm{~mL}$ of water at $0^{\circ} \mathrm{C}$. The $\mathrm{pH}$ was maintained to $6-7$ by adding few drops of a saturated $\mathrm{NaHCO}_{3}$ solution. The mixture was stirred at room temperature for $5 \mathrm{~h}$. A solution of $\mathrm{NaBH}_{4}$ $(2.00 \mathrm{~g}, 52.9 \mathrm{mmol})$ in $20 \mathrm{~mL}$ of water was added dropwise at $0^{\circ} \mathrm{C}$. The mixture was stirred at $\mathrm{rt}$ for $30 \mathrm{~min}$, and neutralized with acetic acid. The precipitate formed was filtered, and rinsed with ethyl acetate. The filtrate was extracted with ethyl acetate $(3 \times 50 \mathrm{~mL})$, the organic phases were washed with $1 \mathrm{~N} \mathrm{Na}_{2} \mathrm{~S}_{2} \mathrm{O}_{3}(75 \mathrm{~mL})$ and with brine $\left(75 \mathrm{~mL}\right.$ ) and dried over $\mathrm{MgSO}_{4}$. The diol 9 was concentrated under vacuum and purified by flash chromatography (cyclohexane/AcOEt: 3/7). A white solid (7.24 g) was isolated in $92 \%$ yield.
The spectral data agreed with those already described. ${ }^{5}$

5.1.2. 2,4-O-Benzylidene-1,3-O -sulfonyl-D-erythritol 10 . To a solution of diol $9(3.00 \mathrm{~g}, 14.3 \mathrm{mmol})$ and anhydrous $\mathrm{NEt}_{3}(5.4 \mathrm{~mL}, 38.6 \mathrm{mmol})$ in $50 \mathrm{~mL}$ of anhydrous DCM at $0^{\circ} \mathrm{C}$ under $\mathrm{Ar}$, was added dropwise a solution of freshly distilled $\mathrm{SOCl}_{2}(1.4 \mathrm{~mL}, 18.6 \mathrm{mmol})$ in $50 \mathrm{~mL}$ of anhydrous DCM. After complete addition $(1 \mathrm{~h})$, the mixture was concentrated under vacuum to give a brown solid. To a solution of this solid $(14.3 \mathrm{mmol})$ in $100 \mathrm{~mL}$ of DCM/ $\mathrm{CH}_{3} \mathrm{CN}$ (5/5) containing $\mathrm{RuCl}_{3}(59.3 \mathrm{mg}, 0.28 \mathrm{mmol})$ was added a solution of $\mathrm{NaIO}_{4}(9.17 \mathrm{~g}, 42.9 \mathrm{mmol})$ in $50 \mathrm{~mL}$ of water. The mixture was stirred for $3 \mathrm{~h}$ and then diluted with $200 \mathrm{~mL}$ of DCM. The aqueous phase was extracted with $50 \mathrm{~mL}$ of DCM. The organic phases were washed with brine $(200 \mathrm{~mL})$, and dried over $\mathrm{MgSO}_{4}$. The sulfate 10 was concentrated under vacuum and purified by flash chromatography (cyclohexane/AcOEt: $9 / 1+0.1 \% \mathrm{NEt}_{3}$ ). A white solid $(3.11 \mathrm{~g})$ was isolated in $80 \%$ yield. $R_{\mathrm{f}} 0.17$ (cyclohexane/AcOEt $9 / 1+0.1 \% \mathrm{NEt}_{3}$ ).

The spectral data agreed with those already described. ${ }^{5}$

5.1.3. 1,3-O-Benzylidene-2,4-O-isopropylidene-L-erythritol 11. A solution of diol $9(4.37 \mathrm{~g}, 20.8 \mathrm{mmol})$, distilled 2-methoxypropene $(6.0 \mathrm{~mL}, 62.9 \mathrm{mmol})$ and TsOH $(67.4 \mathrm{mg}, 0.35 \mathrm{mmol})$ in non-anhydrous DMF was vigorously stirred at $0{ }^{\circ} \mathrm{C}$ for $24 \mathrm{~h}$. The mixture was neutralized with $1.03 \mathrm{~g}$ of $\mathrm{Na}_{2} \mathrm{CO}_{3}$. The precipitate was filtered, and washed with cyclohexane. To the filtrate was added $400 \mathrm{~mL}$ of water, and the aqueous phase was extracted with $200 \mathrm{~mL}$ of cyclohexane. The organic phases were washed with brine, dried over $\mathrm{MgSO}_{4}$ and concentrated under vacuum. The crude product was purified by flash chromatography (cyclohexane/AcOEt: $9 / 1+0.1 \%$ $\mathrm{NEt}_{3}$ ) to give 11 in $96 \%$ yield as a white solid (4.99 g). $\mathrm{R}_{\mathrm{f}}$ 0.39 (cyclohexane/AcOEt $\left.9 / 1+0.1 \% \mathrm{NEt}_{3}\right) \cdot[\alpha]_{\mathrm{D}}=+2(\mathrm{c}$ $\left.1.2, \mathrm{CHCl}_{3}\right) .{ }^{1} \mathrm{H} \mathrm{NMR}\left(\mathrm{CDCl}_{3}\right) \delta 7.80-7.47\left(\mathrm{~m}, 2 \mathrm{H}, \mathrm{H}_{\text {arom }}\right)$; $7.38-7.35\left(\mathrm{~m}, 3 \mathrm{H}, \mathrm{H}_{\mathrm{arom}}\right) ; 5.62\left(\mathrm{~s}, 1 \mathrm{H}, \mathrm{H}_{5}\right) ; 4.23(\mathrm{dd}, 1 \mathrm{H}$, $\left.\mathrm{H}_{4 \mathrm{a}}, J=4.0,10.3 \mathrm{~Hz}\right) ; 4.01-3.95\left(\mathrm{~m}, 2 \mathrm{H}, \mathrm{H}_{1 \mathrm{a}}\right.$ and $\left.\mathrm{H}_{1 \mathrm{~b}}\right)$; $3.93-3.88\left(\mathrm{~m}, 1 \mathrm{H}, \mathrm{H}_{3}\right) ; 3.80-3.73\left(\mathrm{~m}, 2 \mathrm{H}, \mathrm{H}_{4 \mathrm{~b}}\right.$ and $\left.\mathrm{H}_{2}\right) ; 1.57$ $\left(\mathrm{s}, 3 \mathrm{H}, \mathrm{CH}_{3}\right) ; 1.43\left(\mathrm{~s}, 3 \mathrm{H}, \mathrm{CH}_{3}\right) .{ }^{13} \mathrm{C} \mathrm{NMR}\left(\mathrm{CDCl}_{3}\right) \delta 137.2$ $\left(\mathrm{C}_{\text {arom }}\right) ; 129.2-128.3-126.1\left(\mathrm{C}_{\text {arom }}\right) ; 102.0\left(\mathrm{C}_{5}\right) ; 100.0\left(\mathrm{C}_{6}\right)$; $75.0\left(\mathrm{C}_{2}\right) ; 69.6\left(\mathrm{C}_{4}\right) ; 66.6\left(\mathrm{C}_{3}\right) ; 62.3\left(\mathrm{C}_{1}\right) ; 29.1\left(\mathrm{CH}_{3}\right) ; 19.3$ $\left(\mathrm{CH}_{3}\right)$. HRMS (ESI+) calcd for $[\mathrm{M}+\mathrm{Na}]^{+} 273.1103$. Found 273.1113.

5.1.4. 2,4-O-Isopropylidene-L-erythritol 12 . To a solution of $11(4.86 \mathrm{~g}, 19.4 \mathrm{mmol})$ in $160 \mathrm{~mL}$ of ethanol, was added $2.46 \mathrm{~g}$ of $10 \% \mathrm{Pd} / \mathrm{C}$. The mixture was stirred at rt under an atmosphere of $\mathrm{H}_{2}$ (balloon) for 3 days. The catalyst was filtered on membrane and rinsed with methanol. The filtrate was concentrated under vacuum and ethanol $(160 \mathrm{~mL})$ and fresh $10 \% \mathrm{Pd} / \mathrm{C}(2.02 \mathrm{~g})$ were added. The mixture was stirred at rt for 5 days under an atmosphere of $\mathrm{H}_{2}$. The catalyst was filtered on membrane and rinsed with methanol. The filtrate was concentrated under vacuum and the crude product was purified by flash chromatography (cyclohexane/AcOEt: 4/6). A colorless oil was obtained in $88 \%$ yield $(2.78 \mathrm{~g}) . R_{\mathrm{f}} 0.26$ (cyclohexane/AcOEt $4 / 6$ ). $[\alpha]_{\mathrm{D}}=+45(c 1.1, \mathrm{MeOH}) .{ }^{1} \mathrm{H}$ NMR $\left(\mathrm{CD}_{3} \mathrm{OD}\right) \delta 3.82-$ $3.76\left(\mathrm{~m}, 2 \mathrm{H}, \mathrm{H}_{1 \mathrm{a}}\right.$ and $\left.\mathrm{H}_{4 \mathrm{a}}\right) ; 3.69-3.57\left(\mathrm{~m}, 3 \mathrm{H}, \mathrm{H}_{3}, \mathrm{H}_{1 \mathrm{~b}}\right.$ and $\left.\mathrm{H}_{4 \mathrm{~b}}\right) ; 3.48$ (td, $\left.1 \mathrm{H}, \mathrm{H}_{2}, J=5.6,9.2,9.2 \mathrm{~Hz}\right) ; 1.48$ (s, $3 \mathrm{H}$, 
$\left.\mathrm{CH}_{3}\right) ; 1.35\left(\mathrm{~s}, 3 \mathrm{H}, \mathrm{CH}_{3}\right) .{ }^{13} \mathrm{C} \mathrm{NMR}\left(\mathrm{CD}_{3} \mathrm{OD}\right) \delta 99.9\left(\mathrm{C}_{5}\right)$; $76.5\left(\mathrm{C}_{3}\right) ; 65.5\left(\mathrm{C}_{1}\right) ; 64.0\left(\mathrm{C}_{2}\right) ; 63.3\left(\mathrm{C}_{4}\right) ; 28.9\left(\mathrm{CH}_{3}\right) ; 19.7$ $\left(\mathrm{CH}_{3}\right)$. HRMS (ESI+) calcd for $[\mathrm{M}+\mathrm{Na}]^{+} 185.0790$. Found 185.0786.

5.1.5. 2,4-O-Isopropylidene-1,3-O-sulfonyl-L-erythritol 13. To a solution of diol $12(1.08 \mathrm{~g}, 6.66 \mathrm{mmol})$ and anhydrous $\mathrm{NEt}_{3}(2.8 \mathrm{~mL}, 20.1 \mathrm{mmol})$ in $28 \mathrm{~mL}$ of anhydrous $\mathrm{DCM}$ at $0{ }^{\circ} \mathrm{C}$ under $\mathrm{Ar}$, was added dropwise a solution of freshly distilled $\mathrm{SOCl}_{2}(680 \mu \mathrm{L}, 9.37 \mathrm{mmol})$ in $28 \mathrm{~mL}$ of anhydrous DCM. After complete addition (45 min), the mixture was concentrated under vacuum to give a brown solid. To a solution of this solid $(6.6 \mathrm{mmol})$ in $50 \mathrm{~mL}$ of $\mathrm{DCM} / \mathrm{CH}_{3} \mathrm{CN}(5 / 5)$ containing $\mathrm{RuCl}_{3}(33.5 \mathrm{mg}$, $0.16 \mathrm{mmol})$ was added a solution of $\mathrm{NaIO}_{4}(4.64 \mathrm{~g}$, $21.7 \mathrm{mmol}$ ) in $25 \mathrm{~mL}$ of water. The mixture was stirred for $24 \mathrm{~h}$ and then diluted with $100 \mathrm{~mL}$ of DCM and $100 \mathrm{~mL}$ of water. The aqueous phase was extracted twice with $100 \mathrm{~mL}$ of DCM. The organic phases were washed with brine $(2 \times 100 \mathrm{~mL})$, and dried over $\mathrm{MgSO}_{4}$. The sulfate 13 was concentrated under vacuum and purified by flash chromatography (pentane/ether 9/1). A white solid (1.03 g) was isolated in $69 \%$ yield. $R_{\mathrm{f}} 0.16$ (pentane/ether 9/1). $[\alpha]_{\mathrm{D}}=-4\left(\mathrm{c} 1.1, \mathrm{CHCl}_{3}\right) .{ }^{1} \mathrm{H} \mathrm{NMR}\left(\mathrm{CDCl}_{3}\right) \delta 4.66(\mathrm{td}, 1 \mathrm{H}$, $\left.\mathrm{H}_{2}, J_{2-1 \mathrm{a}}=5.4 \mathrm{~Hz}, J_{2-1 \mathrm{~b}}=J_{2-3}=10.3 \mathrm{~Hz}\right) ; 4.61$ (dd, $1 \mathrm{H}$, $\left.\mathrm{H}_{4 \mathrm{a}}, J_{4 \mathrm{a}-3}=J_{4 \mathrm{a}-4 \mathrm{~b}}=10.3 \mathrm{~Hz}\right) ; 4.46\left(\mathrm{dd}, 1 \mathrm{H}, \mathrm{H}_{4 \mathrm{~b}}, J_{4 \mathrm{~b}-3}=\right.$ $\left.4.8 \mathrm{~Hz}, J_{4 \mathrm{~b}-4 \mathrm{a}}=10.3 \mathrm{~Hz}\right) ; 4.22\left(\mathrm{td}, 1 \mathrm{H}, \mathrm{H}_{3}, J_{3-4 \mathrm{~b}}=4.8 \mathrm{~Hz}\right.$, $\left.J_{3-4 \mathrm{a}}=J_{3-2}=10.3 \mathrm{~Hz}\right) ; 4.03\left(\mathrm{dd}, 1 \mathrm{H}, \mathrm{H}_{1 \mathrm{a}}, J_{1 \mathrm{a}-2}=5.4 \mathrm{~Hz}\right.$, $\left.J_{1 \mathrm{a}-1 \mathrm{~b}}=10.3 \mathrm{~Hz}\right) ; 3.92\left(\mathrm{dd}, 1 \mathrm{H}, \mathrm{H}_{1 \mathrm{~b}}, J_{1 \mathrm{~b}-2}=J_{1 \mathrm{~b}-1 \mathrm{a}}=\right.$ $10.3 \mathrm{~Hz}) ; 1.55\left(\mathrm{~s}, 3 \mathrm{H}, \mathrm{CH}_{3}\right) ; 1.43\left(\mathrm{~s}, 3 \mathrm{H}, \mathrm{CH}_{3}\right) .{ }^{13} \mathrm{C} \mathrm{NMR}$ $\left(\mathrm{CDCl}_{3}\right) \delta 101.1\left(\mathrm{C}_{5}\right) ; 76.6\left(\mathrm{C}_{2}\right) ; 73.3\left(\mathrm{C}_{4}\right) ; 64.7\left(\mathrm{C}_{3}\right) ; 60.8$ $\left(\mathrm{C}_{1}\right) ; 28.5\left(\mathrm{CH}_{3}\right) ; 18.9\left(\mathrm{CH}_{3}\right)$. HRMS (ESI+) calcd for $[\mathrm{M}+\mathrm{Na}]^{+}$247.0252. Found 247.0264.

\subsection{General procedure for the coupling reactions}

The thiaheterocycle $(1 \mathrm{mmol})$ and cyclic sulfate $\mathbf{1 0}$ or $\mathbf{1 3}$ $(1.2 \mathrm{mmol})$ were dissolved in 1,1,1,3,3,3-hexafluoroisopropanol HFIP $(3 \mathrm{~mL})$ under argon in the presence of anhydrous $\mathrm{Na}_{2} \mathrm{CO}_{3}(0.24 \mathrm{mmol})$. The mixture was refluxed for the time indicated in Table 1, concentrated under vacuum and purified by flash chromatography (mixture of $\mathrm{DCM} / \mathrm{MeOH}$, see $R_{\mathrm{f}}$ details for each compound).

5.2.1. 1-[(1,4-A nhydro-1-thioerythritol)-1-ium]-1,3dideoxy-2,4-O-isopropylidene-L-erythritol-3-sulfate 20 . $43 \mathrm{mg}$ as a white solid, mixture of non-separable diastereoisomers 20a/20b (83/17). $R_{\mathrm{f}} 0.11$ (DCM/MeOH, 85/15).

Major isomer. ${ }^{1} \mathrm{H}$ NMR $\left(\mathrm{CD}_{3} \mathrm{OD}\right) \delta 4.48-4.45\left(\mathrm{~m}, 2 \mathrm{H}, \mathrm{H}_{2}\right.$ and $\left.\mathrm{H}_{3}\right) ; 4.37-4.23\left(\mathrm{~m}, 2 \mathrm{H}, \mathrm{H}_{2^{\prime}}\right.$ and $\left.\mathrm{H}_{3^{\prime}}\right) ; 4.16\left(\mathrm{dd}, 1 \mathrm{H}, \mathrm{H}_{1^{\prime} \mathrm{a}}\right.$, $\left.J_{1^{\prime} \mathrm{a}-2^{\prime}}=3.4 \mathrm{~Hz}, J_{1^{\prime} \mathrm{a}-1^{\prime} \mathrm{b}}=13.6 \mathrm{~Hz}\right) ; 4.07\left(\mathrm{dd}, 1 \mathrm{H}, \mathrm{H}_{4^{\prime} \mathrm{a}}\right.$, $\left.J_{4^{\prime} \mathrm{a}-3^{\prime}}=5.3 \mathrm{~Hz}, J_{4^{\prime} \mathrm{a}-4^{\prime} \mathrm{b}}=11.5 \mathrm{~Hz}\right), 3.99\left(\mathrm{dd}, 1 \mathrm{H}, \mathrm{H}_{1^{\prime} \mathrm{b}}\right.$, $\left.J_{1^{\prime} \mathrm{b}-2^{\prime}}=4.6 \mathrm{~Hz}, J_{1^{\prime} \mathrm{b}-1^{\prime} \mathrm{a}}=13.6 \mathrm{~Hz}\right), 3.85\left(\mathrm{dd}, 1 \mathrm{H}, \mathrm{H}_{4^{\prime} \mathrm{b}}\right.$, $\left.J_{4^{\prime} \mathrm{b}-3^{\prime}}=9.0 \mathrm{~Hz}, J_{4^{\prime} \mathrm{b}-4^{\prime} \mathrm{a}}=11.5 \mathrm{~Hz}\right), 3.75\left(\mathrm{dd}, 2 \mathrm{H}, \mathrm{H}_{1 \mathrm{a}}\right.$ and $\left.\mathrm{H}_{4 \mathrm{a}}, J=5.0,12.9 \mathrm{~Hz}\right), 3.41-3.36\left(\mathrm{~m}, 2 \mathrm{H}, \mathrm{H}_{1 \mathrm{~b}}\right.$ and $\left.\mathrm{H}_{4 \mathrm{~b}}\right), 1.53$ $\left(\mathrm{s}, 3 \mathrm{H}, \mathrm{CH}_{3}\right), 1.41\left(\mathrm{~s}, 3 \mathrm{H}, \mathrm{CH}_{3}\right) .{ }^{13} \mathrm{C} \mathrm{NMR}\left(\mathrm{CD}_{3} \mathrm{OD}\right) \delta 101.2$ $\left(\mathrm{C}_{5^{\prime}}\right) ; 75.6\left(\mathrm{C}_{2}\right.$ or $\left.\mathrm{C}_{3}\right) ; 75.1\left(\mathrm{C}_{2}\right.$ or $\left.\mathrm{C}_{3}\right) ; 70.9\left(\mathrm{C}_{2^{\prime}}\right.$ or $\left.\mathrm{C}_{3^{\prime}}\right) ; 70.4$ $\left(\mathrm{C}_{2^{\prime}}\right.$ or $\left.\mathrm{C}_{3^{\prime}}\right) ; 63.2\left(\mathrm{C}_{4^{\prime}}\right) ; 50.7\left(\mathrm{C}_{1^{\prime}}\right) ; 46.1\left(\mathrm{C}_{1}\right.$ or $\left.\mathrm{C}_{4}\right) ; 45.6\left(\mathrm{C}_{1}\right.$ or $\left.\mathrm{C}_{4}\right) ; 28.5\left(\mathrm{CH}_{3}\right) ; 19.6\left(\mathrm{CH}_{3}\right)$.

Minor isomer. ${ }^{1} \mathrm{H}$ NMR $\left(\mathrm{CD}_{3} \mathrm{OD}\right) \delta 4.62-4.57\left(\mathrm{~m}, 2 \mathrm{H}, \mathrm{H}_{2}\right.$ 
and $\left.\mathrm{H}_{3}\right) ; 4.37-4.23\left(\mathrm{~m}, 2 \mathrm{H}, \mathrm{H}_{2^{\prime}}\right.$ and $\left.\mathrm{H}_{3^{\prime}}\right) ; 4.08$ (dd, $1 \mathrm{H}, \mathrm{H}_{1^{\prime} \mathrm{a}}$, $J=5.3,11.5 \mathrm{~Hz}$ ); 3.92 (dd, $1 \mathrm{H}, \mathrm{H}_{4^{\prime} \mathrm{a}}, J=3.5,13.4 \mathrm{~Hz}$ ); 3.87-3.81 (m, 2H, $\mathrm{H}_{4^{\prime} \mathrm{b}}$ and $\left.\mathrm{H}_{1}{ }^{\prime} \mathrm{b}\right) ; 3.66-3.55\left(\mathrm{~m}, 4 \mathrm{H}, 2 \mathrm{H}_{1}\right.$ and $\left.2 \mathrm{H}_{4}\right) ; 1.53\left(\mathrm{~s}, 3 \mathrm{H}, \mathrm{CH}_{3}\right) ; 1.41\left(\mathrm{~s}, 3 \mathrm{H}, \mathrm{CH}_{3}\right) .{ }^{13} \mathrm{C} \mathrm{RMN}$ $\left(\mathrm{CD}_{3} \mathrm{OD}\right) \delta 74.7\left(\mathrm{C}_{2}\right.$ or $\left.\mathrm{C}_{3}\right) ; 74.5\left(\mathrm{C}_{2}\right.$ or $\left.\mathrm{C}_{3}\right) ; 70.6\left(\mathrm{C}_{2^{\prime}}\right.$ or $\left.\mathrm{C}_{3^{\prime}}\right) ; 47.9\left(\mathrm{C}_{1}\right.$ or $\left.\mathrm{C}_{4}\right) ; 45.8\left(\mathrm{C}_{1}\right.$ or $\left.\mathrm{C}_{4}\right) ; 28.4\left(\mathrm{CH}_{3}\right) ; 19.7$ $\left(\mathrm{CH}_{3}\right)$.

Remarks for the minor isomer. Absent $\delta$ for some carbons means that the signals are not detected.

5.2.2. 1-[(1,4-Anhydro-1-thioerythritol $)$-1-ium $]-2,4-O$ benzylidene-1,3-dideoxy-D-erythritol-3-sulfate 22 . $148 \mathrm{mg}$ as white solid, mixture of non-separable diastereoisomers 22a/22b (83/17). $R_{\mathrm{f}} 0.22$ (DCM/MeOH, 80/20); mp $135^{\circ} \mathrm{C} ;[\alpha]_{\mathrm{D}}=-45(c 0.91, \mathrm{MeOH})$. IR $\mathrm{KBr} \mathrm{cm}^{-1} 3403$; $1403 ; 1264 ; 1233 ; 1094 ; 1013$.

Major isomer. ${ }^{1} \mathrm{H}$ NMR $\left(\mathrm{CD}_{3} \mathrm{OD}\right) \delta 7.49-7.45(\mathrm{~m}, 2 \mathrm{H}$, $\left.\mathrm{H}_{\text {arom }}\right) ; 7.40-7.36\left(\mathrm{~m}, 3 \mathrm{H}, \mathrm{H}_{\text {arom }}\right) ; 5.68\left(\mathrm{~s}, 1 \mathrm{H}, \mathrm{H}_{5^{\prime}}\right)$; 4.52-4.47 $\left(\mathrm{m}, 1 \mathrm{H}, \mathrm{H}_{4^{\prime} \mathrm{a}}\right) ; 4.45-4.40\left(\mathrm{~m}, 3 \mathrm{H}, \mathrm{H}_{3^{\prime}}, \mathrm{H}_{2}\right.$ and $\left.\mathrm{H}_{3}\right)$; 4.39-4.34 (m, $\left.1 \mathrm{H}, \mathrm{H}_{2^{\prime}}\right) ; 4.29$ (dd, $1 \mathrm{H}, \mathrm{H}_{1^{\prime} \mathrm{a}}, J_{1^{\prime} \mathrm{a}-2^{\prime}}=$ $\left.3.4 \mathrm{~Hz}, J_{1^{\prime} \mathrm{a}-1 \mathrm{~b}}=13.9 \mathrm{~Hz}\right) ; 4.10\left(\mathrm{dd}, 1 \mathrm{H}, \mathrm{H}_{1^{\prime} \mathrm{b}}, J_{1^{\prime} \mathrm{b}-2^{\prime}}=\right.$ $\left.5.1 \mathrm{~Hz}, J_{1^{\prime} \mathrm{b}-1^{\prime} \mathrm{a}}=13.9 \mathrm{~Hz}\right) ; 3.83\left(\mathrm{dd}, 1 \mathrm{H}, \mathrm{H}_{4^{\prime} \mathrm{b}}, J=9.6\right.$, $9.6 \mathrm{~Hz}) ; 3.71\left(\mathrm{dd}, 1 \mathrm{H}, \mathrm{H}_{4 \mathrm{a}}\right.$ or $\left.\mathrm{H}_{1 \mathrm{a}}, J=5.1,11.6 \mathrm{~Hz}\right) ; 3.69$ (dd, $1 \mathrm{H}, \mathrm{H}_{\mathrm{la}}$ or $\left.\mathrm{H}_{4 \mathrm{a}}, J=5.6,10.9 \mathrm{~Hz}\right) ; 3.40\left(\mathrm{dd}, 1 \mathrm{H}, \mathrm{H}_{4 \mathrm{~b}}\right.$ or $\left.\mathrm{H}_{1 \mathrm{~b}}, J=5.1,13.4 \mathrm{~Hz}\right) ; 3.35\left(\mathrm{dd}, 1 \mathrm{H}, \mathrm{H}_{1 \mathrm{~b}}\right.$ or $\mathrm{H}_{4 \mathrm{~b}}, J=5.6$, $13.4 \mathrm{~Hz}) .{ }^{13} \mathrm{C}$ NMR $\left(\mathrm{CD}_{3} \mathrm{OD}\right) \delta 138.4\left(\mathrm{C}_{\text {arom }}\right) ; 130.3-$ $129.3-127.3\left(\mathrm{C}_{\text {arom }}\right) ; 102.7\left(\mathrm{C}_{5^{\prime}}\right) ; 77.4\left(\mathrm{C}_{2^{\prime}}\right) ; 75.7\left(\mathrm{C}_{2}\right.$ or $\left.\mathrm{C}_{3}\right) ; 75.2\left(\mathrm{C}_{2}\right.$ or $\left.\mathrm{C}_{3}\right) ; 69.9\left(\mathrm{C}_{4^{\prime}}\right) ; 69.0\left(\mathrm{C}_{3^{\prime}}\right) ; 50.2\left(\mathrm{C}_{1^{\prime}}\right) ; 46.0$ $\left(\mathrm{C}_{1}\right.$ or $\left.\mathrm{C}_{4}\right) ; 45.8\left(\mathrm{C}_{1}\right.$ or $\left.\mathrm{C}_{4}\right)$

Minor isomer. ${ }^{1} \mathrm{H}$ NMR $\left(\mathrm{CD}_{3} \mathrm{OD}\right) \delta 7.49-7.45(\mathrm{~m}, 2 \mathrm{H}$, $\left.\mathrm{H}_{\text {arom }}\right) ; 7.40-7.36\left(\mathrm{~m}, 3 \mathrm{H}, \mathrm{H}_{\text {arom }}\right) ; 5.68\left(\mathrm{~s}, 1 \mathrm{H}, \mathrm{H}_{5^{\prime}}\right) ; 4.55-$ $4.60\left(\mathrm{~m}, 2 \mathrm{H}, \mathrm{H}_{2}\right.$ and $\left.\mathrm{H}_{3}\right) ; 4.52-4.47\left(\mathrm{~m}, 2 \mathrm{H}, \mathrm{H}_{4 \mathrm{a}^{\prime}}\right.$ and $\left.\mathrm{H}_{3^{\prime}}\right)$; $4.39-4.34\left(\mathrm{~m}, 1 \mathrm{H}, \mathrm{H}_{2^{\prime}}\right) ; 4.04\left(\mathrm{dd}, 1 \mathrm{H}, \mathrm{H}_{1^{\prime} \mathrm{a}}, J_{1^{\prime} \mathrm{a}-2^{\prime}}=3.2 \mathrm{~Hz}\right.$, $\left.J_{1^{\prime} \mathrm{a}-1^{\prime} \mathrm{b}}=13.5 \mathrm{~Hz}\right) ; 3.94\left(\mathrm{dd}, 1 \mathrm{H}, \mathrm{H}_{1^{\prime} \mathrm{b}}, J_{1^{\prime} \mathrm{b}-2^{\prime}}=5.9 \mathrm{~Hz}\right.$, $\left.J_{1^{\prime} \mathrm{b}-1^{\prime} \mathrm{a}}=13.5 \mathrm{~Hz}\right) ; 3.87-3.79\left(\mathrm{~m}, 1 \mathrm{H}, \mathrm{H}_{4^{\prime} \mathrm{b}}\right) ; 3.65-3.51(\mathrm{~m}$, $4 \mathrm{H}, 2 \mathrm{H}_{1}$ and $\left.2 \mathrm{H}_{4}\right) .{ }^{13} \mathrm{C} \mathrm{NMR}\left(\mathrm{CD}_{3} \mathrm{OD}\right) \delta 77.2\left(\mathrm{C}_{2}\right) ; 74.7$ $\left(\mathrm{C}_{2}\right.$ or $\left.\mathrm{C}_{3}\right) ; 74.5\left(\mathrm{C}_{2}\right.$ or $\left.\mathrm{C}_{3}\right) ; 69.4\left(\mathrm{C}_{3^{\prime}}\right) ; 47.3\left(\mathrm{C}_{1}\right.$ or $\left.\mathrm{C}_{4}\right) ; 45.9$ $\left(\mathrm{C}_{1}\right.$ or $\left.\mathrm{C}_{4}\right)$

Remarks for the minor isomer. Absent $\delta$ for some carbons means that the signals are not detected.

5.2.3. 1-[(1,4-Anhydro-1-thiothreitol)-1-ium]-1,3-dideoxy-2,4-O-isopropylidene-L-erythritol-3-sulfate 24 . $259 \mathrm{mg}$ as white solid, mixture of non-separable diastereoisomers 24a/24b (50/50). $R_{\mathrm{f}} 0.31$ (DCM/MeOH, 80/20); $[\alpha]_{\mathrm{D}}=+43(c 1.06, \mathrm{MeOH}) . \mathrm{IR} \mathrm{KBr} \mathrm{cm}^{-1} 3404 ; 1385 ;$ $1268 ; 1228 ; 1050 ; 1013$. For the mixture ${ }^{1} \mathrm{H}$ NMR $\left(\mathrm{CD}_{3} \mathrm{OD}\right)$ $\delta 4.65$ (br s, $2 \mathrm{H}, \mathrm{H}_{2}$ and $\left.\mathrm{H}_{3}\right) ; 4.61$ (br s, $2 \mathrm{H}, \mathrm{H}_{2}$ and $\mathrm{H}_{3}$ ); $4.36-4.25\left(\mathrm{~m}, 4 \mathrm{H}, 2 \mathrm{H}_{3^{\prime}}\right.$ and $\left.2 \mathrm{H}_{2^{\prime}}\right) ; 4.10\left(\mathrm{dd}, 1 \mathrm{H}, \mathrm{H}_{4^{\prime} \mathrm{a}}, J=\right.$ $3.7,5.2 \mathrm{~Hz}) ; 4.08-4.02\left(\mathrm{~m}, 2 \mathrm{H}, \mathrm{H}_{4^{\prime} \mathrm{a}}\right.$ and $\left.\mathrm{H}_{1^{\prime} \mathrm{a}}\right) ; 3.96(\mathrm{br} \mathrm{d}$, $\left.2 \mathrm{H}, 2 \mathrm{H}_{1^{\prime}}, J=4.0 \mathrm{~Hz}\right) ; 3.90-3.72\left(\mathrm{~m}, 9 \mathrm{H}, 2 \mathrm{H}_{1}, 2 \mathrm{H}_{4} . \mathrm{H}_{\mathrm{la}}, \mathrm{H}_{4 \mathrm{a}}\right.$, $\mathrm{H}_{1^{\prime} \mathrm{b}}$ and $2 \mathrm{H}_{4^{\prime} \mathrm{b}}$ ); 3.51 (br d, $1 \mathrm{H}, \mathrm{H}_{4 \mathrm{~b}}$ or $\mathrm{H}_{1 \mathrm{~b}}, J=4.5 \mathrm{~Hz}$ ); 3.48 (br d, $1 \mathrm{H}, \mathrm{H}_{1 \mathrm{~b}}$ or $\left.\mathrm{H}_{4 \mathrm{~b}}, J=4.8 \mathrm{~Hz}\right) ; 1.54\left(\mathrm{~s}, 3 \mathrm{H}, \mathrm{CH}_{3}\right) ; 1.53$ (s, $\left.3 \mathrm{H}, \mathrm{CH}_{3}\right) ; 1.42\left(\mathrm{~s}, 6 \mathrm{H}, \mathrm{CH}_{3}\right) .{ }^{13} \mathrm{C} \mathrm{NMR}\left(\mathrm{CD}_{3} \mathrm{OD}\right) \delta 79.0\left(\mathrm{C}_{2}\right.$ or $\left.\mathrm{C}_{3}\right) ; 78.9\left(\mathrm{C}_{2}\right.$ or $\left.\mathrm{C}_{3}\right) ; 78.7\left(\mathrm{C}_{2}\right.$ or $\left.\mathrm{C}_{3}\right) ; 78.6\left(\mathrm{C}_{2}\right.$ or $\left.\mathrm{C}_{3}\right) ; 70.9$ $\left(\mathrm{C}_{2^{\prime}}\right.$ or $\left.\mathrm{C}_{3^{\prime}}\right) ; 70.7\left(\mathrm{C}_{2^{\prime}}\right.$ or $\left.\mathrm{C}_{3^{\prime}}\right) ; 70.6\left(\mathrm{C}_{2^{\prime}}\right.$ or $\left.\mathrm{C}_{3^{\prime}}\right) ; 63.3\left(\mathrm{C}_{4^{\prime}}\right)$; $53.4\left(\mathrm{C}_{1}\right.$ or $\left.\mathrm{C}_{4}\right) ; 52.5\left(\mathrm{C}_{1}\right.$ or $\left.\mathrm{C}_{4}\right) ; 50.2\left(\mathrm{C}_{1^{\prime}}\right) ; 49.4\left(\mathrm{C}_{1^{\prime}}\right) ; 49.2$ $\left(\mathrm{C}_{1}\right.$ and $\left.\mathrm{C}_{4}\right) ; 28.5\left(\mathrm{CH}_{3}\right) ; 19.6\left(\mathrm{CH}_{3}\right)$.
Remarks. Absent $\delta$ for some carbons means that the signals are not detected.

5.2.4. 1-[(1,4-Anhydro-1-thiothreitol)-1-ium]-2,4-O-benzylidene-1,3-dideoxy-D-erythritol-3-sulfate $26.243 \mathrm{mg}$ as white solid, mixture of non-separable diastereoisomers $26 \mathrm{a} /$ 26b (50/50). $R_{\mathrm{f}} 0.20(\mathrm{DCM} / \mathrm{MeOH}, 85 / 15) ; \mathrm{mp} 143^{\circ} \mathrm{C}$; $[\alpha]_{\mathrm{D}}=-42(c 1.27, \mathrm{MeOH}) . \mathrm{IR} \mathrm{KBr} \mathrm{cm}^{-1} 3397 ; 1402$; $1263 ; 1229 ; 1091 ; 1053 ; 1013$. For the mixture ${ }^{1} \mathrm{H}$ NMR $\left(\mathrm{CD}_{3} \mathrm{OD}\right) \delta 7.51-7.47\left(\mathrm{~m}, 4 \mathrm{H}, \mathrm{H}_{\text {arom }}\right) ; 7.42-7.33(\mathrm{~m}, 6 \mathrm{H}$, $\left.\mathrm{H}_{\text {arom }}\right) ; 5.68\left(\mathrm{~s}, 2 \mathrm{H}, 2 \mathrm{H}_{5^{\prime}}\right) ; 4.63\left(\mathrm{br} \mathrm{s}, 2 \mathrm{H}, \mathrm{H}_{2}\right.$ and $\left.\mathrm{H}_{3}\right) ; 4.59$ (br s, $2 \mathrm{H}, \mathrm{H}_{2}$ and $\left.\mathrm{H}_{3}\right) ; 4.53-4.47\left(\mathrm{~m}, 2 \mathrm{H}, 2 \mathrm{H}_{4^{\prime} \mathrm{a}}\right) ; 4.47-4.40$ $\left(\mathrm{m}, 2 \mathrm{H}, 2 \mathrm{H}_{3^{\prime}}\right) ; 4.38-4.34\left(\mathrm{~m}, 2 \mathrm{H}, 2 \mathrm{H}_{2^{\prime}}\right) ; 4.18\left(\mathrm{dd}, 1 \mathrm{H}, \mathrm{H}_{1^{\prime} \mathrm{a}}\right.$, $\left.J_{1^{\prime} \mathrm{a}-2^{\prime}}=3.3 \mathrm{~Hz}, J_{1^{\prime} \mathrm{a}-1^{\prime} \mathrm{b}}=13.6 \mathrm{~Hz}\right) ; 4.03-4.12(\mathrm{~m}, 2 \mathrm{H}$, $\left.2 \mathrm{H}_{1^{\prime}}\right) ; 3.98\left(\mathrm{dd}, 1 \mathrm{H}, \mathrm{H}_{1^{\prime} \mathrm{b}}, J_{1^{\prime} \mathrm{b}-2^{\prime}}=5.6 \mathrm{~Hz}, J_{1^{\prime} \mathrm{b}-1^{\prime} \mathrm{a}}=\right.$ $13.6 \mathrm{~Hz}) ; 3.86-3.76\left(\mathrm{~m}, 6 \mathrm{H}, 2 \mathrm{H}_{4} . \mathrm{H}_{1 \mathrm{a}}, \mathrm{H}_{4 \mathrm{a}}\right.$ and $\left.2 \mathrm{H}_{4^{\prime} \mathrm{b}}\right)$; 3.74-3.70 (m, 2H, 2 $\left.\mathrm{H}_{1}\right) ; 3.51$ (br d, $1 \mathrm{H}, \mathrm{H}_{4 \mathrm{~b}}$ or $\mathrm{H}_{1 \mathrm{~b}}, J=$ $13.9 \mathrm{~Hz}$ ); 3.47 (br d, $1 \mathrm{H}, \mathrm{H}_{1 \mathrm{~b}}$ or $\mathrm{H}_{4 \mathrm{~b}}, J=13.9 \mathrm{~Hz}$ ). ${ }^{13} \mathrm{C}$ NMR. $\left(\mathrm{CD}_{3} \mathrm{OD}\right) \delta 138.4\left(\mathrm{C}_{\text {arom }}\right) 130.3-129.4-127.3\left(\mathrm{C}_{\text {arom }}\right)$; $102.8\left(\mathrm{C}_{5^{\prime}}\right) ; 102.7\left(\mathrm{C}_{5^{\prime}}\right) ; 79.0\left(\mathrm{C}_{2}\right.$ or $\left.\mathrm{C}_{3}\right) ; 78.9\left(\mathrm{C}_{2}\right.$ or $\left.\mathrm{C}_{3}\right)$; $78.8\left(\mathrm{C}_{2}\right.$ or $\left.\mathrm{C}_{3}\right) ; 78.6\left(\mathrm{C}_{2}\right.$ or $\left.\mathrm{C}_{3}\right) ; 77.5\left(\mathrm{C}_{2^{\prime}}\right) ; 77.3\left(\mathrm{C}_{2^{\prime}}\right) ; 69.9$ $\left(\mathrm{C}_{4^{\prime}}\right) ; 69.1\left(\mathrm{C}_{3^{\prime}}\right) ; 53.4\left(\mathrm{C}_{1}\right.$ or $\left.\mathrm{C}_{4}\right) ; 52.8\left(\mathrm{C}_{1}\right.$ or $\left.\mathrm{C}_{4}\right) ; 49.6\left(\mathrm{C}_{1^{\prime}}\right)$; $49.4\left(\mathrm{C}_{1}, \mathrm{C}_{4}\right.$ or $\left.\mathrm{C}_{1^{\prime}}\right) ; 49.3\left(\mathrm{C}_{1}, \mathrm{C}_{4}\right.$ or $\left.\mathrm{C}_{1}\right) ; 49.1\left(\mathrm{C}_{1}, \mathrm{C}_{4}\right.$ or $\mathrm{C}_{1^{\prime}}$ ).

Remarks. Absent $\delta$ for some carbons means that the signals are not detected.

5.2.5. 1-[(1,5-Anhydro-1-thioxylitol $)$-1-ium $]-2,4-O$-benzylidene-1,3-dideoxy-D-erythritol-3-sulfate $28.65 \mathrm{mg}$ as a white solid, mixture of non-separable diastereoisomers $28 \mathrm{a} / 28 \mathrm{~b}(70 / 30) . R_{\mathrm{f}} 0.18$ (DCM/MeOH, 85/15); $\mathrm{mp} 142{ }^{\circ} \mathrm{C}$; $[\alpha]_{\mathrm{D}}=-46(c 1.02, \mathrm{MeOH}) . \mathrm{IR} \mathrm{KBr} \mathrm{cm}^{-1} 3406 ; 1403$; $1260 ; 1225 ; 1094 ; 1068 ; 1013$.

Major isomer. ${ }^{1} \mathrm{H}$ NMR $\left(\mathrm{CD}_{3} \mathrm{OD}\right) \delta 7.50-7.45(\mathrm{~m}, 2 \mathrm{H}$, $\left.\mathrm{H}_{\text {arom }}\right) ; 7.41-7.34\left(\mathrm{~m}, 3 \mathrm{H}, \mathrm{H}_{\text {arom }}\right) ; 5.69\left(\mathrm{~s}, 1 \mathrm{H}, \mathrm{H}_{5^{\prime}}\right) ; 4.51-$ $4.38\left(\mathrm{~m}, 3 \mathrm{H}, \mathrm{H}_{2^{\prime}}, \mathrm{H}_{3^{\prime}}\right.$ and $\left.\mathrm{H}_{4^{\prime}}\right) ; 4.21-4.16\left(\mathrm{~m}, 2 \mathrm{H}, \mathrm{H}_{2}\right.$ and $\left.\mathrm{H}_{4}\right) ; 4.01\left(\mathrm{dd}, 1 \mathrm{H}, \mathrm{H}_{1^{\prime} \mathrm{a}}, J_{1^{\prime} \mathrm{a}-2^{\prime}}=3.2 \mathrm{~Hz}, J_{1^{\prime} \mathrm{a}-1^{\prime} \mathrm{b}}=13.9 \mathrm{~Hz}\right)$; $3.91\left(\mathrm{dd}, 1 \mathrm{H}, \mathrm{H}_{1^{\prime} \mathrm{b},} J_{1^{\prime} \mathrm{b}-2^{\prime}}=4.9 \mathrm{~Hz}, J_{1^{\prime} \mathrm{b}-1^{\prime} \mathrm{a}}=13.9 \mathrm{~Hz}\right)$; $3.88-3.79\left(\mathrm{~m}, 1 \mathrm{H}, \mathrm{H}_{4^{\prime} \mathrm{b}}\right) ; 3.75\left(\mathrm{dd}, 1 \mathrm{H}, \mathrm{H}_{3}, J=5.1\right.$, $5.1 \mathrm{~Hz}$ ); 3.70 (dd, $1 \mathrm{H}, \mathrm{H}_{5 \mathrm{a}}$ or $\mathrm{H}_{1 \mathrm{a}}, J=2.3,13.1 \mathrm{~Hz}$ ); 3.67 (dd, $1 \mathrm{H}, \mathrm{H}_{1 \mathrm{a}}$ or $\left.\mathrm{H}_{5 \mathrm{a}}, J=2.5,13.1 \mathrm{~Hz}\right) ; 3.52-3.47(\mathrm{~m}, 2 \mathrm{H}$, $\mathrm{H}_{\mathrm{lb}}$ and $\left.\mathrm{H}_{5 \mathrm{~b}}\right) .{ }^{13} \mathrm{C}$ NMR $\delta\left(\mathrm{CD}_{3} \mathrm{OD}\right) \delta 138.3\left(\mathrm{C}_{\mathrm{arom}}\right) ; 130.4$ $129.4-127.3\left(\mathrm{C}_{\text {arom }}\right) ; 102.8\left(\mathrm{C}_{5^{\prime}}\right) ; 77.0\left(\mathrm{C}_{2^{\prime}}\right) ; 69.9\left(\mathrm{C}_{3}\right) ; 69.8$ $\left(\mathrm{C}_{4^{\prime}}\right) ; 69.2\left(\mathrm{C}_{3^{\prime}}\right) ; 68.7\left(\mathrm{C}_{2}\right.$ and $\left.\mathrm{C}_{4}\right) ; 43.8\left(\mathrm{C}_{1^{\prime}}\right) ; 42.5\left(\mathrm{C}_{1}\right.$ or $\left.\mathrm{C}_{5}\right) ; 41.7\left(\mathrm{C}_{1}\right.$ or $\left.\mathrm{C}_{5}\right)$.

Minor isomer. ${ }^{1} \mathrm{H}$ NMR $\left(\mathrm{CD}_{3} \mathrm{OD}\right) \delta 7.50-7.45(\mathrm{~m}, 2 \mathrm{H}$, $\left.\mathrm{H}_{\text {arom }}\right) ; 7.41-7.34\left(\mathrm{~m}, 3 \mathrm{H}, \mathrm{H}_{\text {arom }}\right) ; 5.69\left(\mathrm{~s}, 1 \mathrm{H}, \mathrm{H}_{5^{\prime}}\right) ; 4.51-$ $4.38\left(\mathrm{~m}, 3 \mathrm{H}, \mathrm{H}_{2^{\prime}}, \mathrm{H}_{3^{\prime}}\right.$ and $\left.\mathrm{H}_{4^{\prime} \mathrm{a}}\right) ; 4.12-4.04\left(\mathrm{~m}, 2 \mathrm{H}, 2 \mathrm{H}_{1^{\prime}}\right)$; 3.98-3.89 (m, 2H, $\mathrm{H}_{2}$ and $\left.\mathrm{H}_{4}\right) ; 3.88-3.79\left(\mathrm{~m}, 1 \mathrm{H}, \mathrm{H}_{4^{\prime} \mathrm{b}}\right)$; $3.62-3.56\left(\mathrm{~m}, 3 \mathrm{H}, \mathrm{H}_{1 \mathrm{a}}, \mathrm{H}_{5 \mathrm{a}}\right.$ and $\left.\mathrm{H}_{3}\right) ; 3.40-3.28\left(\mathrm{~m}, 2 \mathrm{H}, \mathrm{H}_{\mathrm{lb}}\right.$ and $\left.\mathrm{H}_{5 \mathrm{~b}}\right) .{ }^{13} \mathrm{C}$ NMR $\delta\left(\mathrm{CD}_{3} \mathrm{OD}\right) \delta 77.1\left(\mathrm{C}_{2^{\prime}}\right) ; 68.9\left(\mathrm{C}_{2}\right.$ or $\left.\mathrm{C}_{4}\right)$; $68.5\left(\mathrm{C}_{2}\right.$ or $\left.\mathrm{C}_{4}\right) ; 45.8\left(\mathrm{C}_{1}^{\prime}\right) ; 40.3\left(\mathrm{C}_{1}\right.$ or $\left.\left.\mathrm{C}_{5}\right)\right) ; 39.7\left(\mathrm{C}_{1}\right.$ or $\left.\mathrm{C}_{5}\right)$.

Remarks for the minor isomer. Absent $\delta$ for some carbons means that the signals are not detected.

5.2.6. 1-[(1,5-Anhydro-1-thioribitol )-1-ium ]-2,4-O-benzylidene-1,3-dideoxy-D-erythritol-3-sulfate 30. Flash chromatography eluent (DCM/MeOH, 85/15), $63 \mathrm{mg}$ as a white solid, mixture of non-separable diastereoisomers 
30a/30b (67/33). $R_{\mathrm{f}} 0.15(\mathrm{DCM} / \mathrm{MeOH}, 80 / 20) ; \mathrm{mp}$ decomp.; $[\alpha]_{\mathrm{D}}=-38(c 0.25, \mathrm{NaOH} 0.01 \mathrm{~N})$. IR $\mathrm{KBr}$ $\mathrm{cm}^{-1} 3450 ; 3388 ; 1407 ; 1250 ; 1231 ; 1098 ; 1014$.

Major isomer. ${ }^{1} \mathrm{H}$ NMR $\left(\mathrm{CD}_{3} \mathrm{OD}\right) \delta 7.50-7.45(\mathrm{~m}, 2 \mathrm{H}$, $\left.\mathrm{H}_{\text {arom }}\right) ; 7.41-7.36\left(\mathrm{~m}, 3 \mathrm{H}, \mathrm{H}_{\text {arom }}\right) ; 5.69\left(\mathrm{~s}, 1 \mathrm{H}, \mathrm{H}_{5^{\prime}}\right) ; 4.51-$ $4.38\left(\mathrm{~m}, 3 \mathrm{H}, \mathrm{H}_{2}{ }^{\prime}, \mathrm{H}_{3^{\prime}}\right.$ and $\left.\mathrm{H}_{4^{\prime} \mathrm{a}}\right) ; 4.27-4.19\left(\mathrm{~m}, 2 \mathrm{H}, \mathrm{H}_{2}\right.$ and $\left.\mathrm{H}_{4}\right) ; 4.08-3.96\left(\mathrm{~m}, 2 \mathrm{H}, \mathrm{H}_{3}\right.$ and $\left.\mathrm{H}_{1^{\prime} \mathrm{a}}\right) ; 3.88-3.81\left(\mathrm{~m}, 2 \mathrm{H}, \mathrm{H}_{1^{\prime} \mathrm{b}}\right.$ and $\left.\mathrm{H}_{4} \mathrm{~b}\right) ; 3.49-3.32\left(\mathrm{~m}, 2 \mathrm{H}, \mathrm{H}_{1 \mathrm{a}}\right.$ and $\left.\mathrm{H}_{5 \mathrm{a}}\right) ; 3.31-3.28(\mathrm{~m}$, $1 \mathrm{H}, \mathrm{H}_{5 \mathrm{~b}}$ or $\mathrm{H}_{1 \mathrm{~b}}$ ); 3.23 (dd, $1 \mathrm{H}, \mathrm{H}_{1 \mathrm{~b}}$ or $\mathrm{H}_{5 \mathrm{~b}}, J=2.7,13.7 \mathrm{~Hz}$ ). ${ }^{13} \mathrm{C}$ NMR $\left(\mathrm{CD}_{3} \mathrm{OD}\right) \delta 138.3\left(\mathrm{C}_{\text {arom }}\right) ; 130.4-129.4-127.3$ $\left(\mathrm{C}_{\text {arom }}\right) ; 102.8\left(\mathrm{C}_{5^{\prime}}\right) ; 77.1\left(\mathrm{C}_{2^{\prime}}\right) ; 71.8\left(\mathrm{C}_{3}\right) ; 69.9\left(\mathrm{C}_{4^{\prime}}\right) ; 69.0$ $\left(\mathrm{C}_{3^{\prime}}\right) ; 66.9\left(\mathrm{C}_{2}\right.$ or $\left.\mathrm{C}_{4}\right) ; 66.7\left(\mathrm{C}_{2}\right.$ or $\left.\mathrm{C}_{4}\right) ; 40.4\left(\mathrm{C}_{1^{\prime}}\right) ; 35.5\left(\mathrm{C}_{1}\right.$ and $\mathrm{C}_{5}$ ).

Minor isomer. ${ }^{1} \mathrm{H}$ NMR $\left(\mathrm{CD}_{3} \mathrm{OD}\right) \delta 7.50-7.45(\mathrm{~m}, 2 \mathrm{H}$, $\left.\mathrm{H}_{\text {arom }}\right) ; 7.41-7.36\left(\mathrm{~m}, 3 \mathrm{H}, \mathrm{H}_{\text {arom }}\right) ; 5.69\left(\mathrm{~s}, 1 \mathrm{H}, \mathrm{H}_{5^{\prime}}\right) ; 4.51-$ $4.38\left(\mathrm{~m}, 3 \mathrm{H}, \mathrm{H}_{2^{\prime}}, \mathrm{H}_{3^{\prime}}\right.$ and $\left.\mathrm{H}_{4^{\prime} \mathrm{a}}\right) ; 4.15$ (dd, $1 \mathrm{H}, \mathrm{H}_{1^{\prime} \mathrm{a}}, J=3.2$, $13.9 \mathrm{~Hz}) ; 4.08-3.96\left(\mathrm{~m}, 4 \mathrm{H}, \mathrm{H}_{2}, \mathrm{H}_{3}, \mathrm{H}_{4}\right.$ and $\left.\mathrm{H}_{1^{\prime} \mathrm{b}}\right) ; 3.88-3.81$ $\left(\mathrm{m}, 1 \mathrm{H}, \mathrm{H}_{4^{\prime} \mathrm{b}}\right) ; 3.49-3.32\left(\mathrm{~m}, 4 \mathrm{H}, 2 \mathrm{H}_{1}\right.$ and $\left.2 \mathrm{H}_{5}\right) .{ }^{13} \mathrm{C} \mathrm{NMR}$ $\left(\mathrm{CD}_{3} \mathrm{OD}\right) \delta 77.0\left(\mathrm{C}_{2^{\prime}}\right) ; 71.9\left(\mathrm{C}_{3}\right) ; 69.1\left(\mathrm{C}_{3^{\prime}}\right) ; 67.6\left(\mathrm{C}_{2}\right.$ or $\left.\mathrm{C}_{4}\right)$; $67.5\left(\mathrm{C}_{2}\right.$ or $\left.\mathrm{C}_{4}\right) ; 46.8\left(\mathrm{C}_{1}\right) ; 38.3\left(\mathrm{C}_{1}\right.$ or $\left.\mathrm{C}_{5}\right) ; .36 .9\left(\mathrm{C}_{1}\right.$ or $\left.\mathrm{C}_{5}\right)$.

Remarks for the minor isomer. Absent $\delta$ for some carbons means that the signals are not detected.

5.2.7. 1-[(1,5-Anhydro-1-deoxy-1-thio-D-arabinitol)-1ium]-2,4-O-benzylidene-1,3-dideoxy-D-erythritol-3-sulfate $32.58 \mathrm{mg}$ as a white solid, mixture of non-separable diastereoisomers 32a/32b (50/50). $R_{\mathrm{f}} 0.19(\mathrm{DCM} / \mathrm{MeOH}$, $85 / 15) ; \mathrm{mp} 141^{\circ} \mathrm{C} ;[\alpha]_{\mathrm{D}}=-48(c 0.44$. MeOH). IR KBr $\mathrm{cm}^{-1} 3404 ; 1402 ; 1263 ; 1227 ; 1091 ; 1013$. For the mixture ${ }^{1} \mathrm{H}$ NMR $\left(\mathrm{CD}_{3} \mathrm{OD}\right) \delta$ 7.50-7.47 (m, 4H, $\left.\mathrm{H}_{\text {arom }}\right) ; 7.42-7.37$ $\left(\mathrm{m}, 6 \mathrm{H}, \mathrm{H}_{\mathrm{arom}}\right) ; 5.68\left(\mathrm{~s}, 1 \mathrm{H}, \mathrm{H}_{5^{\prime}}\right) ; 5.67\left(\mathrm{~s}, 1 \mathrm{H}, \mathrm{H}_{5^{\prime}}\right)$; $4.52-4.36\left(\mathrm{~m}, 7 \mathrm{H}, 2 \mathrm{H}_{2}, \mathrm{H}_{4}\right.$ or $\mathrm{H}_{2}, 2 \mathrm{H}_{4^{\prime} \mathrm{a}}$ and $\left.2 \mathrm{H}_{3^{\prime}}\right)$; 4.34-4.24 (m, 3H, $\mathrm{H}_{2}, \mathrm{H}_{4}, \mathrm{H}_{2}$ or $\left.\mathrm{H}_{4}\right) ; 4.18$ (dd, $1 \mathrm{H}$, $\left.\mathrm{H}_{1^{\prime} \mathrm{a}}, \quad J_{1^{\prime} \mathrm{a}-2^{\prime}}=3.6 \mathrm{~Hz}, J_{1^{\prime} \mathrm{a}-1^{\prime} \mathrm{b}}=14.4 \mathrm{~Hz}\right) ; 4.09(\mathrm{dd}, 1 \mathrm{H}$, $\left.\mathrm{H}_{1^{\prime} \mathrm{b}}, J_{1^{\prime} \mathrm{b}-2^{\prime}}=3.9 \mathrm{~Hz}, J_{1^{\prime} \mathrm{b}-1^{\prime} \mathrm{a}}=14.4 \mathrm{~Hz}\right) ; 4.04(\mathrm{dd}, 1 \mathrm{H}$, $\left.\mathrm{H}_{1^{\prime} \mathrm{a}}, J_{1^{\prime} \mathrm{a}-2^{\prime}}=3.3 \mathrm{~Hz}, J_{1^{\prime} \mathrm{a}-1^{\prime} \mathrm{b}}=14.1 \mathrm{~Hz}\right) ; 3.97(\mathrm{dd}, 1 \mathrm{H}$, $\left.\mathrm{H}_{1^{\prime} \mathrm{b}}, J_{1^{\prime} \mathrm{b}-2^{\prime}}=4.6 \mathrm{~Hz}, J_{1^{\prime} \mathrm{b}-1^{\prime} \mathrm{a}}=14.1 \mathrm{~Hz}\right) ; 3.88-3.78(\mathrm{~m}, 4 \mathrm{H}$, $2 \mathrm{H}_{3}$ and $\left.2 \mathrm{H}_{4^{\prime} \mathrm{b}}\right) ; 3.62-3.52\left(\mathrm{~m}, 3 \mathrm{H}, \mathrm{H}_{5 \mathrm{a}}, \mathrm{H}_{1 \mathrm{a}}, \mathrm{H}_{5 \mathrm{a}}\right.$ or $\left.\mathrm{H}_{1 \mathrm{a}}\right)$; $3.47-3.36\left(\mathrm{~m}, 3 \mathrm{H}, \mathrm{H}_{1 \mathrm{~b}}, \mathrm{H}_{5 \mathrm{~b}}, \mathrm{H}_{1 \mathrm{a}}\right.$ or $\left.\mathrm{H}_{5 \mathrm{a}}\right) ; 3.30-3.25(\mathrm{~m}, 1 \mathrm{H}$, $\mathrm{H}_{5 \mathrm{~b}}$ or $\left.\mathrm{H}_{1 \mathrm{~b}}\right) ; 3.23\left(\mathrm{dd}, 1 \mathrm{H}, \mathrm{H}_{1 \mathrm{~b}}\right.$ or $\left.\mathrm{H}_{5 \mathrm{~b}}, J=5.4,13.4 \mathrm{~Hz}\right) .{ }^{13} \mathrm{C}$ $\mathrm{RMN}\left(\mathrm{CD}_{3} \mathrm{OD}\right) \delta 138.3\left(\mathrm{C}_{\text {arom }}\right) ; 130.4-130.3-129.4-127.3$ $\left(\mathrm{C}_{\text {arom }}\right) ; 102.8\left(\mathrm{C}_{5^{\prime}}\right) ; 102.7\left(\mathrm{C}_{5^{\prime}}\right) ; 77.3\left(\mathrm{C}_{2^{\prime}}\right) ; 77.0\left(\mathrm{C}_{2^{\prime}}\right) ; 71.7$ $\left(\mathrm{C}_{3}\right) ; 70.6\left(\mathrm{C}_{3}\right) ; 69.8\left(\mathrm{C}_{4^{\prime}}\right) ; 69.4\left(\mathrm{C}_{2}, \mathrm{C}_{4}\right.$ or $\left.\mathrm{C}_{3^{\prime}}\right) ; 69.1\left(\mathrm{C}_{2}, \mathrm{C}_{4}\right.$ or $\left.\mathrm{C}_{3^{\prime}}\right) ; 68.7\left(\mathrm{C}_{2}, \mathrm{C}_{4}\right.$ or $\left.\mathrm{C}_{3^{\prime}}\right) ; 68.2\left(\mathrm{C}_{2}, \mathrm{C}_{4}\right.$ or $\left.\mathrm{C}_{3^{\prime}}\right) ; 65.1\left(\mathrm{C}_{4}\right.$ or $\left.\mathrm{C}_{2}\right) ; 63.2\left(\mathrm{C}_{4}\right.$ or $\left.\mathrm{C}_{2}\right) ; 45.6\left(\mathrm{C}_{1}^{\prime}\right) ; 43.4\left(\mathrm{C}_{1^{\prime}}\right) ; 42.2\left(\mathrm{C}_{1}\right.$ or $\left.\mathrm{C}_{5}\right)$; $37.8\left(\mathrm{C}_{1}\right.$ or $\left.\mathrm{C}_{5}\right) ; 37.2\left(\mathrm{C}_{1}\right.$ or $\left.\mathrm{C}_{5}\right)$.

Remarks. Absent $\delta$ for some carbons means that the signals are not detected.

5.2.8. 1-[(1,6-Anhydro-1-thio-D-mannitol)-1-ium]-1,3dideoxy-2,4-O-isopropylidene-L-erythritol-3-sulfate 34 . Flash chromatography eluent (DCM/MeOH, 85/15), $23 \mathrm{mg}$ as a white solid. $R_{\mathrm{f}} 0.17(\mathrm{DCM} / \mathrm{MeOH}, 80 / 20) ; \mathrm{mp}$ $168^{\circ} \mathrm{C} ;[\alpha]_{\mathrm{D}}=-9\left(c 1.08, \mathrm{H}_{2} \mathrm{O}\right)$. IR KBr cm${ }^{-1} 3388 ; 3349$; $1390 ; 1276 ; 1225 ; 1091 ; 1059 ; 1012 .{ }^{1} \mathrm{H}$ NMR $\left(\mathrm{D}_{2} \mathrm{O}\right) \delta$ $4.69-4.67\left(\mathrm{~m}, 1 \mathrm{H}, \mathrm{H}_{2}\right.$ or $\left.\mathrm{H}_{5}\right) ; 4.60-4.57\left(\mathrm{~m}, 1 \mathrm{H}, \mathrm{H}_{5}\right.$ or $\mathrm{H}_{2}$ ); 4.40 (ddd, $1 \mathrm{H}, \mathrm{H}_{2^{\prime}}, J_{2^{\prime}-1^{\prime} \mathrm{a}}=3.1 \mathrm{~Hz}, J_{2^{\prime}-1^{\prime} \mathrm{b}}=6.4 \mathrm{~Hz}$, $\left.J_{2^{\prime}-3^{\prime}}=9.1 \mathrm{~Hz}\right) ; 4.37\left(\mathrm{td}, 1 \mathrm{H}, \mathrm{H}_{3^{\prime}}, J_{3^{\prime}-4^{\prime} \mathrm{a}}=5.6 \mathrm{~Hz}, J_{3^{\prime}-4^{\prime} \mathrm{b}}=\right.$ $\left.J_{3^{\prime}-2^{\prime}}=9.1 \mathrm{~Hz}\right) ; 4.11\left(\mathrm{dd}, 1 \mathrm{H}, \quad \mathrm{H}_{4^{\prime} \mathrm{a}}, \quad J_{4^{\prime} \mathrm{a}-3^{\prime}}=5.6 \mathrm{~Hz}\right.$,
$\left.J_{4^{\prime} \mathrm{a}-4^{\prime} \mathrm{b}}=11.9 \mathrm{~Hz}\right) ; 4.08\left(\mathrm{dd}, 1 \mathrm{H}, \mathrm{H}_{1^{\prime} \mathrm{a}}, J_{1^{\prime} \mathrm{a}-2^{\prime}}=3.1 \mathrm{~Hz}\right.$, $\left.J_{1^{\prime} \mathrm{a}-1^{\prime} \mathrm{b}}=14.1 \mathrm{~Hz}\right) ; 4.09-4.03\left(\mathrm{~m}, 1 \mathrm{H}, \mathrm{H}_{6 \mathrm{a}}\right.$ or $\left.\mathrm{H}_{1 \mathrm{a}}\right)$; $3.97\left(\mathrm{dd}, 1 \mathrm{H}, \mathrm{H}_{4^{\prime} \mathrm{b}}, J_{4^{\prime} \mathrm{b}-3^{\prime}}=9.1 \mathrm{~Hz}, J_{4^{\prime} \mathrm{b}-4^{\prime} \mathrm{a}}=11.9 \mathrm{~Hz}\right)$; 3.90-3.73 (m, 5H, $\mathrm{H}_{1 \mathrm{a}}$ or $\mathrm{H}_{6 \mathrm{a}}, \mathrm{H}_{3}, \mathrm{H}_{\mathrm{1}^{\prime} \mathrm{b}}, \mathrm{H}_{4}$ and $\mathrm{H}_{6 \mathrm{~b}}$ or $\left.\mathrm{H}_{1 \mathrm{~b}}\right) ; 3.66-3.62\left(\mathrm{~m}, 1 \mathrm{H}, \mathrm{H}_{1 \mathrm{~b}}\right.$ or $\left.\mathrm{H}_{6 \mathrm{~b}}\right) ; 1.58\left(\mathrm{~s}, 3 \mathrm{H}, \mathrm{CH}_{3}\right) ; 1.46$ $\left(\mathrm{s}, 3 \mathrm{H}, \mathrm{CH}_{3}\right) .{ }^{13} \mathrm{C}$ NMR $\left(\mathrm{D}_{2} \mathrm{O}\right) \delta 100.8\left(\mathrm{C}_{5^{\prime}}\right) ; 74.3\left(\mathrm{C}_{4}\right.$ or $\left.\mathrm{C}_{3}\right)$; $74.2\left(\mathrm{C}_{4}\right.$ or $\left.\mathrm{C}_{3}\right) ; 70.4\left(\mathrm{C}_{3^{\prime}}\right) ; 70.0\left(\mathrm{C}_{2}\right.$ or $\left.\mathrm{C}_{5}\right) ; 68.8\left(\mathrm{C}_{2^{\prime}}\right) ; 66.9$ $\left(\mathrm{C}_{5}\right.$ or $\left.\mathrm{C}_{2}\right) ; 61.6\left(\mathrm{C}_{4^{\prime}}\right) ; 44.8\left(\mathrm{C}_{1}\right) ; 43.7\left(\mathrm{C}_{6}\right.$ or $\left.\mathrm{C}_{1}\right) ; 39.7\left(\mathrm{C}_{1}\right.$ or $\left.\mathrm{C}_{6}\right) ; 26.8\left(\mathrm{CH}_{3}\right) ; 18.8\left(\mathrm{CH}_{3}\right)$.

5.2.9. 1-[(1,6-Anhydro-1-thio-D-mannitol $)-1$-ium $]-2,4-O$ benzylidene-1,3-dideoxy-D-erythritol-3-sulfate 36. Flash chromatography eluent (DCM/MeOH, 85/15), $84 \mathrm{mg}$ as a white solid. $R_{\mathrm{f}} 0.20(\mathrm{DCM} / \mathrm{MeOH}, 80 / 20)$. Mp $160^{\circ} \mathrm{C}$. $[\alpha]_{\mathrm{D}}=-63(c 0.92, \mathrm{MeOH}) . \mathrm{IR} \mathrm{KBr} \mathrm{cm}^{-1} 3387 ; 1414$; $1258 ; 1219 ; 1096 ; 1060 ; 1013 .{ }^{1} \mathrm{H}$ NMR $\delta ;\left(\mathrm{CD}_{3} \mathrm{OD}\right) \delta$ 7.46-7.50 (m, 2H, $\left.\mathrm{H}_{\text {arom }}\right) ; 7.34-7.41\left(\mathrm{~m}, 3 \mathrm{H}, \mathrm{H}_{\text {arom }}\right) ; 5.67(\mathrm{~s}$, $\left.1 \mathrm{H}, \mathrm{H}_{5^{\prime}}\right) ; 4.52-4.58\left(\mathrm{~m}, 1 \mathrm{H}, \mathrm{H}_{2}\right.$ or $\left.\mathrm{H}_{5}\right) ; 4.42-4.50(\mathrm{~m}, 2 \mathrm{H}$, $\mathrm{H}_{3^{\prime}}$ and $\mathrm{H}_{4^{\prime} \mathrm{a}}$ ); 4.40 (td, $1 \mathrm{H}, \mathrm{H}_{5}$ or $\mathrm{H}_{2}, J=1.5,1.5,8.6 \mathrm{~Hz}$ ); $4.33-4.37\left(\mathrm{~m}, 1 \mathrm{H}, \mathrm{H}_{2^{\prime}}\right) ; 4.11\left(\mathrm{dd}, 1 \mathrm{H}, \mathrm{H}_{1^{\prime} \mathrm{a}}, J_{1^{\prime} \mathrm{a}-2^{\prime}}=3.5 \mathrm{~Hz}\right.$, $\left.J_{1^{\prime} \mathrm{a}-1^{\prime} \mathrm{b}}=14.1 \mathrm{~Hz}\right) ; 3.98\left(\mathrm{dd}, 1 \mathrm{H}, \mathrm{H}_{1^{\prime} \mathrm{b}}, J_{1^{\prime} \mathrm{b}-2^{\prime}}=4.8 \mathrm{~Hz}\right.$, $\left.J_{1}{\mathrm{~b}-11^{\prime} \mathrm{a}}^{2}=14.1 \mathrm{~Hz}\right) ; 3.96\left(\mathrm{dd}, 1 \mathrm{H}, \mathrm{H}_{6 \mathrm{a}}\right.$ or $\mathrm{H}_{1 \mathrm{a}}, J=8.5$, $13.1 \mathrm{~Hz}) ; 3.79-3.86\left(\mathrm{~m}, 1 \mathrm{H}, \mathrm{H}_{4^{\prime} \mathrm{b}}\right) ; 3.69-3.76\left(\mathrm{~m}, 3 \mathrm{H}, \mathrm{H}_{3}\right.$, $\mathrm{H}_{4}$ and $\mathrm{H}_{6 \mathrm{~b}}$ or $\left.\mathrm{H}_{1 \mathrm{~b}}\right) ; 3.66\left(\mathrm{dd}, 1 \mathrm{H}, \mathrm{H}_{1 \mathrm{a}}\right.$ or $\mathrm{H}_{6 \mathrm{a}}, J=8.0$, $14.4 \mathrm{~Hz}) ; 3.54\left(\mathrm{dd}, 1 \mathrm{H}, \mathrm{H}_{1 \mathrm{~b}}\right.$ or $\left.\mathrm{H}_{6 \mathrm{~b}}, J=1.2,14.4 \mathrm{~Hz}\right) .{ }^{13} \mathrm{C}$ NMR (CD $\left.{ }_{3} \mathrm{OD}\right) \quad \delta \quad 138.4\left(\mathrm{C}_{\text {arom }}\right) ; 130.3-129.4-127.3$ $\left(\mathrm{C}_{\text {arom }}\right) ; 102.8\left(\mathrm{C}_{5^{\prime}}\right) ; 77.3\left(\mathrm{C}_{2^{\prime}}\right) ; 76.1\left(\mathrm{C}_{4}\right.$ or $\left.\mathrm{C}_{3}\right) ; 75.8\left(\mathrm{C}_{4}\right.$ or $\left.\mathrm{C}_{3}\right) ; 71.2\left(\mathrm{C}_{2}\right.$ or $\left.\mathrm{C}_{5}\right) ; 69.9\left(\mathrm{C}_{4^{\prime}}\right) ; 69.0\left(\mathrm{C}_{2}, \mathrm{C}_{5}\right.$ or $\left.\mathrm{C}_{3^{\prime}}\right) ; 68.3$ $\left(\mathrm{C}_{2}, \mathrm{C}_{5}\right.$ or $\left.\mathrm{C}_{3^{\prime}}\right) ; 47.4\left(\mathrm{C}_{1^{\prime}}\right) ; 46.6\left(\mathrm{C}_{6}\right.$ or $\left.\mathrm{C}_{1}\right) ; 42.0\left(\mathrm{C}_{1}\right.$ or $\left.\mathrm{C}_{6}\right)$.

\subsection{General procedure for isopropylidene and benzylidene protecting groups removal}

Method A. (Conditions (b)). The protected zwitterion $(0.224 \mathrm{mmol})$ and $151 \mathrm{mg}$ of Dowex 50WX8 (16-40 mesh, $\mathrm{H}^{+}$form) in $7 \mathrm{~mL}$ of distilled water were stirred at rt for the time indicated in Table 1. The mixture was filtered, and the resin washed with water. The water was removed under vacuum and the residue was purified by flash chromatography (mixture of $\mathrm{DCM} / \mathrm{MeOH} / \mathrm{H}_{2} \mathrm{O}$, see $R_{\mathrm{f}}$ details for each compound).

Method B. (Conditions (c)). The benzylidene protected zwitterion $(0.237 \mathrm{mmol})$ and $10 \% \mathrm{Pd} / \mathrm{C}(80 \mathrm{mg})$ in $4.5 \mathrm{~mL}$ of $\mathrm{AcOH} / \mathrm{H}_{2} \mathrm{O}(4 / 1)$ were stirred under an atmosphere of $\mathrm{H}_{2}$ (balloon) at $\mathrm{rt}$ for several days. As the reaction slowed down, the catalyst was removed by filtration over a membrane and washed with $\mathrm{MeOH}$ and distilled water. The filtrate was concentrated under vacuum, and $4.5 \mathrm{~mL}$ of $\mathrm{AcOH} / \mathrm{H}_{2} \mathrm{O}(4 / 1)$ and $76 \mathrm{mg}$ of $10 \% \mathrm{Pd} / \mathrm{C}$ were added. The reaction was stirred under an atmosphere of $\mathrm{H}_{2}$ at $\mathrm{rt}$ for the total time indicated in Table 1 . The catalyst was removed by filtration over a membrane and washed with distilled water. The mixture was concentrated under vacuum and purified by flash chromatography (mixture of $\mathrm{DCM} / \mathrm{MeOH} / \mathrm{H}_{2} \mathrm{O}$, see $R_{\mathrm{f}}$ details for each compound).

5.3.1. 1-[(1,4-A nhydro-1-thioerythritol)-1-ium]-1,3dideoxy-L-erythritol-3-sulfate 21. Method A. $56 \mathrm{mg}$ as a colorless oil, mixture of non-separable diastereoisomers 21a/21b (83/17). $R_{\mathrm{f}} 0.26\left(\mathrm{DCM} / \mathrm{MeOH} / \mathrm{H}_{2} \mathrm{O}, 55 / 40 / 5\right)$; $[\alpha]_{\mathrm{D}}=+22\left(c 1.06, \mathrm{H}_{2} \mathrm{O}\right)$. IR KBr cm${ }^{-1} 3398 ; 1253 ; 1234$; $1096 ; 1057 ; 1013 ; 920$. 
Major isomer. ${ }^{1} \mathrm{H}$ NMR $\left(\mathrm{D}_{2} \mathrm{O}\right) \delta 4.62-4.57\left(\mathrm{~m}, 2 \mathrm{H}, \mathrm{H}_{2}\right.$ and $\left.\mathrm{H}_{3}\right) ; 4.30-4.40\left(\mathrm{~m}, 2 \mathrm{H}, \mathrm{H}_{3^{\prime}}\right.$ and $\left.\mathrm{H}_{2^{\prime}}\right) ; 4.07$ (dd, $1 \mathrm{H}, \mathrm{H}_{1^{\prime} \mathrm{a}}, J=$ $3.4,13.6 \mathrm{~Hz}) ; 3.95\left(\mathrm{dd}, 1 \mathrm{H}, \mathrm{H}_{4^{\prime} \mathrm{a}}, J=3.1,9.7 \mathrm{~Hz}\right) ; 3.93-3.89$ $\left(\mathrm{m}, 1 \mathrm{H}, \mathrm{H}_{1^{\prime} \mathrm{b}}\right) ; 3.87-3.80\left(\mathrm{~m}, 3 \mathrm{H}, \mathrm{H}_{1 \mathrm{a}}, \mathrm{H}_{4 \mathrm{a}}\right.$ and $\left.\mathrm{H}_{4^{\prime} \mathrm{b}}\right) ; 3.47$ (td, $2 \mathrm{H}, \mathrm{H}_{\mathrm{bb}}$ and $\mathrm{H}_{4 \mathrm{~b}}, J=5.4,13.2,13.2 \mathrm{~Hz}$ ). ${ }^{13} \mathrm{C}$ NMR $\left(\mathrm{D}_{2} \mathrm{O}\right) \delta 79.7\left(\mathrm{C}_{3^{\prime}}\right) ; 73.4\left(\mathrm{C}_{2}\right.$ or $\left.\mathrm{C}_{3}\right) ; 73.3\left(\mathrm{C}_{2}\right.$ or $\left.\mathrm{C}_{3}\right) ; 65.6$ $\left(\mathrm{C}_{2^{\prime}}\right) ; 59.5\left(\mathrm{C}_{4^{\prime}}\right) ; 51.1\left(\mathrm{C}_{1}{ }^{\prime}\right) ; 44.2\left(\mathrm{C}_{1}\right.$ or $\left.\mathrm{C}_{4}\right) ; 43.8\left(\mathrm{C}_{1}\right.$ or $\left.\mathrm{C}_{4}\right)$.

Minor isomer. ${ }^{1} \mathrm{H}$ NMR $\left(\mathrm{D}_{2} \mathrm{O}\right) \delta 4.75-4.70\left(\mathrm{~m}, 2 \mathrm{H}, \mathrm{H}_{2}\right.$ and $\left.\mathrm{H}_{3}\right) ; 4.40-4.30\left(\mathrm{~m}, 2 \mathrm{H}, \mathrm{H}_{2^{\prime}}\right.$ and $\left.\mathrm{H}_{3^{\prime}}\right) ; 3.89-3.93(\mathrm{~m}, 1 \mathrm{H}$, $\left.\mathrm{H}_{1^{\prime} \mathrm{a}}\right) ; 3.80-3.87\left(\mathrm{~m}, 2 \mathrm{H}, \mathrm{H}_{1^{\prime} \mathrm{b}}\right.$ and $\left.\mathrm{H}_{4^{\prime} \mathrm{a}}\right) ; 3.63-3.77(\mathrm{~m}, 5 \mathrm{H}$, $2 \mathrm{H}_{1}, 2 \mathrm{H}_{4}$ and $\left.\mathrm{H}_{4^{\prime} \mathrm{b}}\right) .{ }^{13} \mathrm{C}$ NMR $\left(\mathrm{D}_{2} \mathrm{O}\right) \delta 79.9\left(\mathrm{C}_{3^{\prime}}\right) ; 72.8\left(\mathrm{C}_{2}\right.$ or $\left.\mathrm{C}_{3}\right) ; 72.7\left(\mathrm{C}_{2}\right.$ or $\left.\mathrm{C}_{3}\right) ; 65.4\left(\mathrm{C}_{2^{\prime}}\right) ; 48.4\left(\mathrm{C}_{1^{\prime}}\right) ; 44.3\left(\mathrm{C}_{1}\right.$ or $\left.\mathrm{C}_{4}\right) ; 43.5\left(\mathrm{C}_{1}\right.$ or $\left.\mathrm{C}_{4}\right)$. HRMS $(\mathrm{ESI}+)$ calcd for $[\mathrm{M}+\mathrm{Na}]^{+}$ 327.0184. Found 327.0186 .

Remarks for the minor isomer. Absent $\delta$ for some carbons means that the signals are not detected.

5.3.2. 1-[(1,4-Anhydro-1-thioery thritol)-1-ium]-1,3dideoxy-D-erythritol-3-sulfate 23. Method B. $23 \mathrm{mg}$ as a colorless oil, mixture of non-separable diastereoisomers 23a/23b (86/14). $R_{\mathrm{f}} 0.25\left(\mathrm{DCM} / \mathrm{MeOH} / \mathrm{H}_{2} \mathrm{O}, 55 / 40 / 5\right)$; $[\alpha]_{\mathrm{D}}=-22\left(c 1.05, \mathrm{H}_{2} \mathrm{O}\right)$; IR KBr cm ${ }^{-1} 3396 ; 1405 ; 1253$; $1231 ; 1095 ; 1013$.

Major isomer. ${ }^{1} \mathrm{H}$ NMR $\left(\mathrm{D}_{2} \mathrm{O}\right) \delta$ i $4.65-4.60\left(\mathrm{~m}, 2 \mathrm{H}, \mathrm{H}_{2}\right.$ and $\left.\mathrm{H}_{3}\right) ; 4.40$ (ddd, $1 \mathrm{H}, \mathrm{H}_{2^{\prime}}, J_{2^{\prime}-1^{\prime} \mathrm{a}}=3.5 \mathrm{~Hz}, J_{2^{\prime}-1^{\prime} \mathrm{b}}=$ $\left.7.5 \mathrm{~Hz}, J_{2^{\prime}-3^{\prime}}=14.8 \mathrm{~Hz}\right) ; 4.38-4.35\left(\mathrm{~m}, 1 \mathrm{H}, \mathrm{H}_{3^{\prime}}\right) ; 4.10(\mathrm{dd}$, $\left.1 \mathrm{H}, \mathrm{H}_{1^{\prime} \mathrm{a}}, J_{1^{\prime} \mathrm{a}-2^{\prime}}=3.5 \mathrm{~Hz}, J_{1^{\prime} \mathrm{a}-1^{\prime} \mathrm{b}}=13.6 \mathrm{~Hz}\right) ; 3.99(\mathrm{dd}, 1 \mathrm{H}$, $\left.\mathrm{H}_{4^{\prime} \mathrm{a}}, J=3.3,12.8 \mathrm{~Hz}\right) ; 3.96\left(\mathrm{dd}, 1 \mathrm{H}, \mathrm{H}_{1^{\prime} \mathrm{b}}, J_{1^{\prime} \mathrm{b}-2^{\prime}}=7.5 \mathrm{~Hz}\right.$, $\left.J_{1^{\prime} \mathrm{b}-1^{\prime} \mathrm{a}}=13.6 \mathrm{~Hz}\right) ; 3.97-3.83\left(\mathrm{~m}, 3 \mathrm{H}, \mathrm{H}_{1 \mathrm{a}}, \mathrm{H}_{4 \mathrm{a}}, \mathrm{H}_{4^{\prime} \mathrm{b}}\right) ; 3.51$ (2dd, $2 \mathrm{H}, \mathrm{H}_{1 \mathrm{~b}}$ and $\mathrm{H}_{4 \mathrm{~b}}, J=5.4,13.3 \mathrm{~Hz}$ ). ${ }^{13} \mathrm{C} \mathrm{NMR}\left(\mathrm{D}_{2} \mathrm{O}\right) \delta$ $79.8\left(\mathrm{C}_{3}\right) ; 73.5\left(\mathrm{C}_{2}\right.$ or $\left.\mathrm{C}_{3}\right) ; 73.4\left(\mathrm{C}_{2}\right.$ or $\left.\mathrm{C}_{3}\right) ; 65.7\left(\mathrm{C}_{2^{\prime}}\right) ; 59.6$ $\left(\mathrm{C}_{4^{\prime}}\right) ; 51.1\left(\mathrm{C}_{1^{\prime}}\right) ; 44.2\left(\mathrm{C}_{1}\right.$ or $\left.\mathrm{C}_{4}\right) ; 43.9\left(\mathrm{C}_{1}\right.$ or $\left.\mathrm{C}_{4}\right)$.

Minor isomer. ${ }^{1} \mathrm{H}$ NMR $\left(\mathrm{D}_{2} \mathrm{O}\right) \delta 4.77-4.73\left(\mathrm{~m}, 2 \mathrm{H}, \mathrm{H}_{2}\right.$ and $\left.\mathrm{H}_{3}\right) ; 4.43-4.38\left(\mathrm{~m}, 2 \mathrm{H}, \mathrm{H}_{2^{\prime}}\right.$ and $\left.\mathrm{H}_{3^{\prime}}\right) ; 3.97-3.83\left(\mathrm{~m}, 3 \mathrm{H}, \mathrm{H}_{4^{\prime} \mathrm{s}}\right.$ and $\left.2 \mathrm{H}_{1^{\prime}}\right) ; 3.82-3.66\left(\mathrm{~m}, 5 \mathrm{H}, 2 \mathrm{H}_{1}, 2 \mathrm{H}_{4}\right.$ and $\left.\mathrm{H}_{4}{ }^{\prime} \mathrm{b}\right) .{ }^{13} \mathrm{C} \mathrm{NMR}$ $\left(\mathrm{D}_{2} \mathrm{O}\right) \delta 72.9\left(\mathrm{C}_{2}\right.$ or $\left.\mathrm{C}_{3}\right) ; 44.4\left(\mathrm{C}_{1}\right.$ or $\left.\mathrm{C}_{4}\right) ; 43.6\left(\mathrm{C}_{1}\right.$ or $\left.\mathrm{C}_{4}\right)$. HRMS (ESI+) calcd for $[\mathrm{M}+\mathrm{Na}]^{+} 327.0184$. Found 327.0182 .

Remarks for the minor isomer. Absent $\delta$ for some carbons means that the signals are not detected.

5.3.3. 1-[(1,4-Anhydro-1-thiothreitol)-1-ium]-1,3-dideoxy-L-erythritol-3-sulfate 25. Method A. $72 \mathrm{mg}$ as a colorless oil, mixture of non-separable diastereoisomers $25 \mathrm{a} / 25 \mathrm{~b}(50 / 50) . R_{\mathrm{f}} 0.52\left(\mathrm{DCM} / \mathrm{MeOH} / \mathrm{H}_{2} \mathrm{O}, 55 / 40 / 5\right)$; $[\alpha]_{\mathrm{D}}=+23\left(c 1.01, \mathrm{H}_{2} \mathrm{O}\right)$. IR KBr cm ${ }^{-1} 3394 ; 1404 ; 1254$; $1229 ; 1053 ; 1011 .{ }^{1} \mathrm{H}$ NMR $\left(\mathrm{D}_{2} \mathrm{O}\right) \delta 4.85\left(\mathrm{br} \mathrm{s}, 2 \mathrm{H}, \mathrm{H}_{2}\right.$ and $\left.\mathrm{H}_{3} ;\right) ; 4.80\left(\right.$ br s, $2 \mathrm{H}, \mathrm{H}_{2}$ and $\left.\mathrm{H}_{3}\right) ; 4.45-4.35\left(\mathrm{~m}, 4 \mathrm{H}, 2 \mathrm{H}_{3^{\prime}}\right.$ and $\left.2 \mathrm{H}_{2^{\prime}}\right)$; 4.06-3.80 (m, $14 \mathrm{H}, 4 \mathrm{H}_{4^{\prime}}, 4 \mathrm{H}_{1^{\prime}}, \mathrm{H}_{1 \mathrm{a}}, \mathrm{H}_{4 \mathrm{a}}, 2 \mathrm{H}_{1}$ and $\left.2 \mathrm{H}_{4}\right) ; 3.68$ (br d, $1 \mathrm{H}, \mathrm{H}_{4 \mathrm{~b}}$ or $\mathrm{H}_{1 \mathrm{~b}}, J=15.4 \mathrm{~Hz}$ ); 3.65 (br d, $1 \mathrm{H}, \mathrm{H}_{1 \mathrm{~b}}$ or $\left.\mathrm{H}_{4 \mathrm{~b}}, J=14.6 \mathrm{~Hz}\right) .{ }^{13} \mathrm{C} \mathrm{NMR}\left(\mathrm{D}_{2} \mathrm{O}\right) \delta 79.9\left(\mathrm{C}_{3^{\prime}}\right)$; $79.7\left(\mathrm{C}_{3^{\prime}}\right) ; 77.2\left(\mathrm{C}_{2}\right.$ or $\left.\mathrm{C}_{3}\right) ; 77.2\left(\mathrm{C}_{2}\right.$ or $\left.\mathrm{C}_{3}\right) ; 77.1\left(\mathrm{C}_{2}\right.$ or $\left.\mathrm{C}_{3}\right)$; $77.0\left(\mathrm{C}_{2}\right.$ or $\left.\mathrm{C}_{3}\right) ; 65.9\left(\mathrm{C}_{2^{\prime}}\right) ; 65.6\left(\mathrm{C}_{2^{\prime}}\right) ; 59.6\left(\mathrm{C}_{4^{\prime}}\right) ; 51.1\left(\mathrm{C}_{1}\right.$, $\mathrm{C}_{4}$ or $\left.\mathrm{C}_{1^{\prime}}\right) ; 50.5\left(\mathrm{C}_{1}, \mathrm{C}_{4}\right.$ or $\left.\mathrm{C}_{1^{\prime}}\right) ; 50.4\left(\mathrm{C}_{1}, \mathrm{C}_{4}\right.$ or $\left.\mathrm{C}_{1^{\prime}}\right) ; 50.2$ $\left(\mathrm{C}_{1}, \mathrm{C}_{4}\right.$ or $\left.\mathrm{C}_{1^{\prime}}\right) ; 47.6\left(\mathrm{C}_{1}\right.$ or $\left.\mathrm{C}_{4}\right) ; 47.3\left(\mathrm{C}_{1}\right.$ or $\left.\mathrm{C}_{4}\right)$. HRMS $(\mathrm{ESI}+)$ calcd for $[\mathrm{M}+\mathrm{Na}]^{+} 327.0184$. Found 327.0188 .
Remarks for the minor isomer. Absent $\delta$ for some carbons means that the signals are not detected.

5.3.4. 1-[(1,4-Anhydro-1-thiothreitol)-1-ium]-1,3-dideoxy-D-erythritol-3-sulfate 27. Method B. $52 \mathrm{mg}$ as a colorless oil, mixture of non-separable diastereoisomers $27 \mathrm{a} / 27 \mathrm{~b}(50 / 50) . \quad R_{\mathrm{f}} 0.54\left(\mathrm{DCM} / \mathrm{MeOH} / \mathrm{H}_{2} \mathrm{O}, 55 / 40 / 5\right)$; $[\alpha]_{\mathrm{D}}=-24\left(c 1.04 . \mathrm{H}_{2} \mathrm{O}\right) . \mathrm{IR} \mathrm{KBr} \mathrm{cm}{ }^{-1} 3395 ; 1404 ; 1254$; $1233 ; 1051 ; 1009 .{ }^{1} \mathrm{H}$ NMR $\left(\mathrm{D}_{2} \mathrm{O}\right) \delta 4.82-4.80\left(\mathrm{~m}, 2 \mathrm{H}, \mathrm{H}_{2}\right.$ and $\left.\mathrm{H}_{3}\right) ; 4.77-4.75\left(\mathrm{~m}, 2 \mathrm{H}, \mathrm{H}_{2}\right.$ and $\left.\mathrm{H}_{3}\right) ; 4.41-4.31(\mathrm{~m}, 4 \mathrm{H}$, $2 \mathrm{H}_{2^{\prime}}$ and $\left.2 \mathrm{H}_{3^{\prime}}\right) ; 4.02-3.77\left(\mathrm{~m}, 14 \mathrm{H}, 4 \mathrm{H}_{4^{\prime}}, 4 \mathrm{H}_{1^{\prime}}, \mathrm{H}_{1 \mathrm{a}}, \mathrm{H}_{4 \mathrm{a}}\right.$, $2 \mathrm{H}_{1}$ and $\left.2 \mathrm{H}_{4}\right) ; 3.65$ (br d, $1 \mathrm{H}, \mathrm{H}_{4 \mathrm{~b}}$ or $\mathrm{H}_{1 \mathrm{~b}}, J=14.9 \mathrm{~Hz}$ ); 3.61 (br d, $1 \mathrm{H}, \mathrm{H}_{1 \mathrm{~b}}$ or $\mathrm{H}_{4 \mathrm{~b}}, J=14.7 \mathrm{~Hz}$ ). ${ }^{13} \mathrm{C} \operatorname{NMR}\left(\mathrm{D}_{2} \mathrm{O}\right) \delta 79.9$ $\left(\mathrm{C}_{3^{\prime}}\right) ; 79.7\left(\mathrm{C}_{3^{\prime}}\right) ; 77.2\left(\mathrm{C}_{2}\right.$ or $\left.\mathrm{C}_{3}\right) ; 77.1\left(\mathrm{C}_{2}\right.$ or $\left.\mathrm{C}_{3}\right) ; 77.1\left(\mathrm{C}_{2}\right.$ or $\left.\mathrm{C}_{3}\right) ; 77.0\left(\mathrm{C}_{2}\right.$ or $\left.\mathrm{C}_{3}\right) ; 65.9\left(\mathrm{C}_{2^{\prime}}\right) ; 65.5\left(\mathrm{C}_{2^{\prime}}\right) ; 59.5\left(\mathrm{C}_{4^{\prime}}\right) ; 51.1$ $\left(\mathrm{C}_{1}, \mathrm{C}_{4}\right.$ or $\left.\mathrm{C}_{1^{\prime}}\right) ; 50.5\left(\mathrm{C}_{1}, \mathrm{C}_{4}\right.$ or $\left.\mathrm{C}_{1}\right) ; 50.4\left(\mathrm{C}_{1}, \mathrm{C}_{4}\right.$ or $\left.\mathrm{C}_{1^{\prime}}\right)$; $50.2\left(\mathrm{C}_{1}, \mathrm{C}_{4}\right.$ or $\mathrm{C}_{1}$ ) $) ; 47.5\left(\mathrm{C}_{1}\right.$ or $\left.\mathrm{C}_{4}\right) ; 47.3\left(\mathrm{C}_{1}\right.$ or $\left.\mathrm{C}_{4}\right)$. HRMS $(\mathrm{ESI}+)$ calcd for $[\mathrm{M}+\mathrm{Na}]^{+}$327.0184. Found 327.0179.

5.3.5. 1-[(1,5-Anhydro-1-thioxylitol)-1-ium $]$-1,3-dideoxyD-erythritol-3-sulfate 29. Method A. $24 \mathrm{mg}$ as a colorless oil, mixture of non-separable diastereoisomers $29 \mathrm{a} / 29 \mathrm{~b}$ $(77 / 23) . R_{\mathrm{f}} 0.37\left(\mathrm{DCM} / \mathrm{MeOH} / \mathrm{H}_{2} \mathrm{O}, 55 / 40 / 5\right) ;[\alpha]_{\mathrm{D}}=-14$ (c $1.06, \mathrm{H}_{2} \mathrm{O}$ ). IR KBr cm${ }^{-1} 3397 ; 1403 ; 1250 ; 1063 ; 1009$.

Major isomer. ${ }^{1} \mathrm{H}$ NMR $\left(\mathrm{D}_{2} \mathrm{O}\right) \delta$ 4.53-4.43 (m, $\left.1 \mathrm{H}, \mathrm{H}_{2^{\prime}}\right)$; 4.42-4.36 (m, $\left.1 \mathrm{H}, \mathrm{H}_{3^{\prime}}\right)$; 4.32-4.27 $\left(\mathrm{m}, 2 \mathrm{H}, \mathrm{H}_{4}\right.$ and $\left.\mathrm{H}_{2}\right)$; 4.06-3.95 (m, $\left.1 \mathrm{H}, \mathrm{H}_{4^{\prime} \mathrm{a}}\right) ; 3.92-3.86\left(\mathrm{~m}, 2 \mathrm{H}, \mathrm{H}_{1^{\prime} \mathrm{a}}\right.$ and $\left.\mathrm{H}_{4^{\prime} \mathrm{b}}\right)$; $3.85-3.70\left(\mathrm{~m}, 4 \mathrm{H}, \mathrm{H}_{\mathrm{la}}, \mathrm{H}_{5 \mathrm{a}}, \mathrm{H}_{1^{\prime} \mathrm{b}}\right.$ and $\left.\mathrm{H}_{3}\right) ; 3.61-3.50(\mathrm{~m}, 2 \mathrm{H}$, $\mathrm{H}_{1 \mathrm{~b}}$ and $\left.\mathrm{H}_{5 \mathrm{~b}}\right) .{ }^{13} \mathrm{C} \mathrm{NMR}\left(\mathrm{D}_{2} \mathrm{O}\right) \delta 79.8\left(\mathrm{C}_{3^{\prime}}\right) ; 71.5\left(\mathrm{C}_{3}\right) ; 66.6$ $\left(\mathrm{C}_{4}\right.$ and $\left.\mathrm{C}_{2}\right)$; $65.5\left(\mathrm{C}_{2^{\prime}}\right) ; 59.5\left(\mathrm{C}_{4^{\prime}}\right) ; 42.9\left(\mathrm{C}_{1^{\prime}}\right) ; 38.7\left(\mathrm{C}_{1}\right.$ or $\left.\mathrm{C}_{5}\right) ; 38.5\left(\mathrm{C}_{1}\right.$ or $\left.\mathrm{C}_{5}\right)$.

Minor isomer. ${ }^{1} \mathrm{H}$ NMR $\left(\mathrm{D}_{2} \mathrm{O}\right) \delta$ 4.53-4.43 $\left(\mathrm{m}, 1 \mathrm{H}, \mathrm{H}_{3^{\prime}}\right)$; $4.42-4.36\left(\mathrm{~m}, 1 \mathrm{H}, \mathrm{H}_{2^{\prime}}\right) ; 4.06-3.95\left(\mathrm{~m}, 4 \mathrm{H}, \mathrm{H}_{1^{\prime} \mathrm{a}}, \mathrm{H}_{4^{\prime} \mathrm{a}}, \mathrm{H}_{2}\right.$ and $\left.\mathrm{H}_{4}\right) ; 3.92-3.86\left(\mathrm{~m}, 2 \mathrm{H}, \mathrm{H}_{1^{\prime} \mathrm{b}}\right.$ and $\left.\mathrm{H}_{4} \mathrm{~b}\right) ; 3.85-3.70(\mathrm{~m}, 2 \mathrm{H}$, $\mathrm{H}_{1 \mathrm{a}}$ and $\left.\mathrm{H}_{5 \mathrm{a}}\right) ; 3.61-3.50\left(\mathrm{~m}, 1 \mathrm{H}, \mathrm{H}_{3}\right) ; 3.42\left(\mathrm{br} \mathrm{d}, 1 \mathrm{H}, \mathrm{H}_{5 \mathrm{~b}}\right.$ or $\left.\mathrm{H}_{1 \mathrm{~b}}, J=11.7 \mathrm{~Hz}\right) ; 3.39$ (br d, $1 \mathrm{H}, \mathrm{H}_{\mathrm{lb}}$ or $\mathrm{H}_{5 \mathrm{~b}}, J=11.7 \mathrm{~Hz}$ ). ${ }^{13} \mathrm{C}$ NMR $\left(\mathrm{D}_{2} \mathrm{O}\right) \delta 75.6\left(\mathrm{C}_{3}\right) ; 67.3\left(\mathrm{C}_{4}\right.$ or $\left.\mathrm{C}_{2}\right)$; $65.2\left(\mathrm{C}_{2^{\prime}}\right)$; $47.9\left(\mathrm{C}_{1^{\prime}}\right) ; 40.7\left(\mathrm{C}_{1}\right.$ or $\left.\mathrm{C}_{5}\right) ; 40.6\left(\mathrm{C}_{1}\right.$ or $\left.\mathrm{C}_{5}\right)$. HRMS (ESI+) calcd for $[\mathrm{M}+\mathrm{Na}]+357.0290$. Found 357.0293 .

Remarks for the minor isomer. Absent $\delta$ for some carbons means that the signals are not detected.

5.3.6. 1-[(1,5-Anhydro-1-thioribitol)-1-ium]-1,3-dideoxyD-erythritol-3-sulfate 31. Method A. $24 \mathrm{mg}$ as a colorless oil, mixture of non-separable diastereoisomers $31 \mathrm{a} / \mathbf{3 1 b}$ $(80 / 20) . R_{\mathrm{f}} 0.16\left(\mathrm{DCM} / \mathrm{MeOH} / \mathrm{H}_{2} \mathrm{O}, 55 / 40 / 5\right) .[\alpha]_{\mathrm{D}}=-19$ (c $\left.0.51, \mathrm{H}_{2} \mathrm{O}\right)$. IR KBr cm${ }^{-1} 3403 ; 1403 ; 1254 ; 1231 ; 1089$; 1016.

Major isomer. ${ }^{1} \mathrm{H}$ NMR $\left(\mathrm{D}_{2} \mathrm{O}\right) \delta 4.50-4.34\left(\mathrm{~m}, 4 \mathrm{H}, \mathrm{H}_{3^{\prime}}, \mathrm{H}_{4}\right.$. $\mathrm{H}_{2}$ and $\left.\mathrm{H}_{2^{\prime}}\right) ; 4.10-4.08\left(\mathrm{~m}, 1 \mathrm{H}, \mathrm{H}_{3}\right) ; 4.00\left(\mathrm{dd}, 1 \mathrm{H}, \mathrm{H}_{4^{\prime} \mathrm{a}}\right.$, $\left.J_{4^{\prime} \mathrm{a}-3^{\prime}}=3.3 \mathrm{~Hz}, J_{4^{\prime} \mathrm{a}-4^{\prime} \mathrm{b}}=12.8 \mathrm{~Hz}\right) ; 3.89\left(\mathrm{dd}, 1 \mathrm{H}, \mathrm{H}_{4^{\prime} \mathrm{b}}\right.$, $\left.J_{4^{\prime} \mathrm{b}-3^{\prime}}=2.9 \mathrm{~Hz}, J_{4^{\prime} \mathrm{b}-4^{\prime} \mathrm{a}}=12.8 \mathrm{~Hz}\right) ; 3.83\left(\mathrm{dd}, 1 \mathrm{H}, \mathrm{H}_{1^{\prime} \mathrm{a}}\right.$, $\left.J_{1^{\prime} \mathrm{a}-2^{\prime}}=3.4 \mathrm{~Hz}, J_{1^{\prime} \mathrm{a}-1^{\prime} \mathrm{b}}=13.8 \mathrm{~Hz}\right) ; 3.70\left(\mathrm{dd}, 1 \mathrm{H}, \mathrm{H}_{1^{\prime} \mathrm{b}}\right.$, $\left.J_{1^{\prime} \mathrm{b}-2^{\prime}}=7.7 \mathrm{~Hz}, J_{1^{\prime} \mathrm{b}-1^{\prime} \mathrm{a}}=13.8 \mathrm{~Hz}\right) ; 3.61-3.52(\mathrm{~m}, 2 \mathrm{H}$, $\mathrm{H}_{1 \mathrm{a}}$ and $\left.\mathrm{H}_{5 \mathrm{a}}\right) ; 3.49-3.40\left(\mathrm{~m}, 2 \mathrm{H}, \mathrm{H}_{1 \mathrm{~b}}\right.$ and $\left.\mathrm{H}_{5 \mathrm{~b}}\right) .{ }^{13} \mathrm{C}$ NMR $\left(\mathrm{D}_{2} \mathrm{O}\right) \delta 79.8\left(\mathrm{C}_{3^{\prime}}\right) ; 69.6\left(\mathrm{C}_{3}\right) ; 65.5\left(\mathrm{C}_{4}, \mathrm{C}_{2}\right.$ or $\left.\mathrm{C}_{2^{\prime}}\right) ; 65.2\left(\mathrm{C}_{4}\right.$ $\mathrm{C}_{2}$, or $\left.\mathrm{C}_{2^{\prime}}\right) ; 59.5\left(\mathrm{C}_{4^{\prime}}\right) ; 41.7\left(\mathrm{C}_{1^{\prime}}\right) ; 35.6\left(\mathrm{C}_{1}\right.$ or $\left.\mathrm{C}_{5}\right) ; 35.5\left(\mathrm{C}_{1}\right.$ or $\mathrm{C}_{5}$ ). 
Minor isomer. ${ }^{1} \mathrm{H}$ NMR $\left(\mathrm{D}_{2} \mathrm{O}\right) \delta 4.50-4.34\left(\mathrm{~m}, 2 \mathrm{H}, \mathrm{H}_{3^{\prime}}\right.$ and $\left.\mathrm{H}_{2^{\prime}}\right) ; 4.20-4.14\left(\mathrm{~m}, 3 \mathrm{H}, \mathrm{H}_{2}, \mathrm{H}_{3}\right.$ and $\left.\mathrm{H}_{4}\right) ; 4.01-3.96(\mathrm{~m}, 2 \mathrm{H}$, $\mathrm{H}_{1^{\prime} \mathrm{a}}$ and $\left.\mathrm{H}_{4^{\prime} \mathrm{a}}\right) ; 3.90-3.86\left(\mathrm{~m}, 2 \mathrm{H}, \mathrm{H}_{1^{\prime} \mathrm{b}}\right.$ and $\left.\mathrm{H}_{4^{\prime} \mathrm{b}}\right) ; 3.61-3.52$ $\left(\mathrm{m}, 2 \mathrm{H}, \mathrm{H}_{1 \mathrm{a}}\right.$ and $\left.\mathrm{H}_{5 \mathrm{a}}\right) ; 3.49-3.40\left(\mathrm{~m}, 2 \mathrm{H}, \mathrm{H}_{1 \mathrm{~b}}\right.$ and $\left.\mathrm{H}_{5 \mathrm{~b}}\right) .{ }^{13} \mathrm{C}$ $\operatorname{NMR}\left(\mathrm{D}_{2} \mathrm{O}\right) \delta 79.8\left(\mathrm{C}_{3^{\prime}}\right) ; 70.1\left(\mathrm{C}_{3}\right) ; 65.8\left(\mathrm{C}_{4}\right.$ and $\left.\mathrm{C}_{2}\right) ; 47.8$ $\left(\mathrm{C}_{1^{\prime}}\right) ; 35.2\left(\mathrm{C}_{1}\right.$ or $\left.\mathrm{C}_{5}\right) ; 34.9\left(\mathrm{C}_{1}\right.$ or $\left.\mathrm{C}_{5}\right)$. HRMS (ESI +$)$ calcd for $[\mathrm{M}+\mathrm{Na}]^{+}$357.0290. Found 357.0289.

Remarks for the minor isomer. Absent $\delta$ for some carbons means that the signals are not detected.

5.3.7. 1-[(1,5-Anhydro-1-thio-D-arabinitol $)-1$-ium $]-1,3-$ dideoxy-D-erythritol-3-sulfate 33. Method A. $25 \mathrm{mg}$ as a colorless oil, mixture of non-separable diastereoisomers 33a/33b (50/50). $R_{\mathrm{f}} 0.40\left(\mathrm{DCM} / \mathrm{MeOH} / \mathrm{H}_{2} \mathrm{O}, 55 / 40 / 5\right)$. $[\alpha]_{\mathrm{D}}=-44\left(c 0.99, \mathrm{H}_{2} \mathrm{O}\right)$. IR $(\mathrm{KBr}) \mathrm{cm}^{-1} 3400,1251$; 1069 ; 1014. ${ }^{1} \mathrm{H}$ NMR $\left(\mathrm{D}_{2} \mathrm{O}\right) \delta 4.58\left(\mathrm{td}, 1 \mathrm{H}, \mathrm{H}_{4}\right.$ or $\mathrm{H}_{2}, J=$ $2.4,2.4,6.8 \mathrm{~Hz}) ; 4.50$ (td, $1 \mathrm{H}, \mathrm{H}_{4}$ or $\mathrm{H}_{2}, J=2.1,6.0$, $6.0 \mathrm{~Hz}) ; 4.47-4.30\left(\mathrm{~m}, 6 \mathrm{H}, 2\left(\mathrm{H}_{2}\right.\right.$ or $\left.\mathrm{H}_{4}\right), 2 \mathrm{H}_{3^{\prime}}$ and $\left.2 \mathrm{H}_{2^{\prime}}\right)$; 4.01-3.86 (m, 9H, 4 $4 \mathrm{H}_{1^{\prime}}, 4 \mathrm{H}_{4^{\prime}}$ and $\left.\mathrm{H}_{3}\right) ; 3.82-3.73(\mathrm{~m}, 3 \mathrm{H}$, $\mathrm{H}_{1 \mathrm{a}}, \mathrm{H}_{3}$ and $\left.\mathrm{H}_{5 \mathrm{a}}\right) ; 3.67$ (dd, $1 \mathrm{H}, \mathrm{H}_{5 \mathrm{a}}$ or $\mathrm{H}_{1 \mathrm{a}}, J=2.3,13.3 \mathrm{~Hz}$ ); 3.60-3.53 (m, 3H, 2( $\mathrm{H}_{5 \mathrm{~b}}$ or $\left.\mathrm{H}_{1 \mathrm{~b}}\right)$ and $\mathrm{H}_{1 \mathrm{a}}$ or $\left.\mathrm{H}_{5 \mathrm{a}}\right) ; 3.51$ (dd, $1 \mathrm{H}, \mathrm{H}_{1 \mathrm{~b}}$ or $\left.\mathrm{H}_{5 \mathrm{~b}}, J=9.7,12.4 \mathrm{~Hz}\right) ; 3.32\left(\mathrm{dd}, 1 \mathrm{H}, \mathrm{H}_{1 \mathrm{~b}}\right.$ or $\mathrm{H}_{5 \mathrm{~b}}$, $J=9.1,12.9 \mathrm{~Hz}) .{ }^{13} \mathrm{C}$ NMR $\left(\mathrm{D}_{2} \mathrm{O}\right) \delta 79.8\left(\mathrm{C}_{3^{\prime}}\right) ; 79.8\left(\mathrm{C}_{3^{\prime}}\right)$; $71.6\left(\mathrm{C}_{3}\right) ; 69.2\left(\mathrm{C}_{3}\right) ; 66.0\left(\mathrm{C}_{4}\right.$ or $\left.\mathrm{C}_{2}\right) ; 65.8\left(\mathrm{C}_{4}\right.$ or $\left.\mathrm{C}_{2}\right) ; 65.3$ $\left(\mathrm{C}_{2^{\prime}}\right) ; 64.9\left(\mathrm{C}_{4}\right.$ or $\left.\mathrm{C}_{2}\right) ; 64.6\left(\mathrm{C}_{4}\right.$ or $\left.\mathrm{C}_{2}\right) ; 59.5\left(\mathrm{C}_{4^{\prime}}\right) ; 46.0\left(\mathrm{C}_{1^{\prime}}\right)$; $45.7\left(\mathrm{C}_{1^{\prime}}\right) ; 40.7\left(\mathrm{C}_{1}\right.$ or $\left.\mathrm{C}_{5}\right) ; 38.9\left(\mathrm{C}_{1}\right.$ or $\left.\mathrm{C}_{5}\right) ; 38.8\left(\mathrm{C}_{1}\right.$ or $\left.\mathrm{C}_{5}\right)$; $36.8\left(\mathrm{C}_{1}\right.$ or $\left.\mathrm{C}_{5}\right)$. HRMS (ESI+) calcd for $[\mathrm{M}+\mathrm{Na}]^{+}$ 357.0290. Found 357.0290.

5.3.8. 1-[(1,6-Anhydro-1-thio-D-mannitol)-1-ium $]-1,3-$ dideoxy-L-erythritol-3-sulfate 35. Method A. $14 \mathrm{mg}$ as a colorless oil. $R_{\mathrm{f}} 0.34$ (DCM/MeOH/ $\left.\mathrm{H}_{2} \mathrm{O}, 55 / 40 / 5\right)$; $[\alpha]_{\mathrm{D}}=-29\left(c 1.19, \mathrm{H}_{2} \mathrm{O}\right)$. IR $(\mathrm{KBr}) \mathrm{cm}^{-1} 3397,1248$, $1103 ; 1056 ; 1019 .{ }^{1} \mathrm{H}$ NMR $\left(\mathrm{D}_{2} \mathrm{O}\right) \delta 4.70-4.68\left(\mathrm{~m}, 1 \mathrm{H}, \mathrm{H}_{2}\right.$ or $\left.\mathrm{H}_{5}\right) ; 4.61-4.58\left(\mathrm{~m}, 1 \mathrm{H}, \mathrm{H}_{2}\right) ; 4.42\left(\mathrm{td}, 1 \mathrm{H}, \mathrm{H}_{5}\right.$ or $\mathrm{H}_{2}, J=3.3$, $7.9,7.9 \mathrm{~Hz}) ; 4.39-4.33\left(\mathrm{~m}, 1 \mathrm{H}, \mathrm{H}_{3^{\prime}}\right) ; 4.15\left(\mathrm{dd}, 1 \mathrm{H}, \mathrm{H}_{1^{\prime} \mathrm{a}}, J=\right.$ $8.8,13.2 \mathrm{~Hz}) ; 4.02\left(\mathrm{dd}, 1 \mathrm{H}, \mathrm{H}_{6 \mathrm{a}}\right.$ or $\mathrm{H}_{1 \mathrm{a}}, J=3.4,10.0 \mathrm{~Hz}$ ); 3.99 (dd, $\left.1 \mathrm{H}, \mathrm{H}_{4^{\prime} \mathrm{b}}, J=3.1,8.6 \mathrm{~Hz}\right) ; 3.91-3.85\left(\mathrm{~m}, 3 \mathrm{H}, \mathrm{H}_{4}\right.$ or $\mathrm{H}_{3}, \mathrm{H}_{1} \mathrm{~b}$ and $\mathrm{H}_{4}{ }^{\prime} \mathrm{b}$ ); 3.80 (dd, $1 \mathrm{H}, \mathrm{H}_{3}$ or $\mathrm{H}_{4 \cdot} J=1.8,8.8 \mathrm{~Hz}$ ); $3.78-3.70\left(\mathrm{~m}, 2 \mathrm{H}, \mathrm{H}_{1 \mathrm{a}}\right.$ or $\mathrm{H}_{6 \mathrm{a}}$ and $\mathrm{H}_{6 \mathrm{~b}}$ or $\left.\mathrm{H}_{1 \mathrm{~b}}\right) ; 3.65-3.60(\mathrm{~m}$, $1 \mathrm{H}, \mathrm{H}_{1 \mathrm{~b}}$ or $\left.\mathrm{H}_{6 \mathrm{~b}}\right) .{ }^{13} \mathrm{C} \mathrm{NMR}\left(\mathrm{D}_{2} \mathrm{O}\right) \delta 79.9\left(\mathrm{C}_{3^{\prime}}\right) ; 74.3\left(\mathrm{C}_{4}\right.$ or $\left.\mathrm{C}_{3}\right) ; 74.1\left(\mathrm{C}_{4}\right.$ or $\left.\mathrm{C}_{3}\right) ; 69.9\left(\mathrm{C}_{2}\right.$ or $\left.\mathrm{C}_{5}\right) ; 66.9\left(\mathrm{C}_{2}{ }^{\prime}\right) ; 65.5\left(\mathrm{C}_{5}\right.$ or $\left.\mathrm{C}_{2}\right) ; 59.5\left(\mathrm{C}_{4^{\prime}}\right) ; 47.4\left(\mathrm{C}_{6}\right.$ or $\left.\mathrm{C}_{1}\right) ; 43.7\left(\mathrm{C}_{1}\right) ; 39.1\left(\mathrm{C}_{1}\right.$ or $\left.\mathrm{C}_{6}\right)$. HRMS $(\mathrm{ESI}+)$ calcd for $[\mathrm{M}+\mathrm{Na}]^{+}$387.0396. Found 387.0396 .

5.3.9. 1-[(1,6-Anhydro-1-thio-D-mannitol)-1-ium $]-1,3-$ dideoxy-D-erythritol-3-sulfate 37. Method A. $11 \mathrm{mg}$ as a colorless oil. $R_{\mathrm{f}} 0.36$ (DCM/MeOH/ $\left.\mathrm{H}_{2} \mathrm{O}, 55 / 40 / 5\right)$. $[\alpha]_{\mathrm{D}}=-70\left(c 0.83, \mathrm{H}_{2} \mathrm{O}\right)$. IR $(\mathrm{KBr}) \mathrm{cm}^{-1} 3404 ; 1405$; $1249 ; 1102 ; 1054 ; 1013 .{ }^{1} \mathrm{H}$ NMR $\left(\mathrm{D}_{2} \mathrm{O}\right) \delta$ $4.69-4.67$ $\left(\mathrm{m}, 1 \mathrm{H}, \mathrm{H}_{2}\right.$ or $\left.\mathrm{H}_{5}\right) ; 4.61-4.58\left(\mathrm{~m}, 1 \mathrm{H}, \mathrm{H}_{2^{\prime}}\right) ; 4.40-4.33(\mathrm{~m}$, $2 \mathrm{H}, \mathrm{H}_{3^{\prime}}$ and $\mathrm{H}_{5}$ or $\mathrm{H}_{2}$ ); 4.13 (dd, $1 \mathrm{H}, \mathrm{H}_{1^{\prime} \mathrm{a}}, J=8.6,13.0 \mathrm{~Hz}$ ); $4.02-3.97\left(\mathrm{~m}, 1 \mathrm{H}, \mathrm{H}_{6 \mathrm{a}}\right.$ or $\left.\mathrm{H}_{1 \mathrm{a}}\right) ; 3.98\left(\mathrm{dd}, 1 \mathrm{H}, \mathrm{H}_{4^{\prime} \mathrm{a}}, J=2.7\right.$, $12.7 \mathrm{~Hz}) ; 3.89-3.75\left(\mathrm{~m}, 5 \mathrm{H}, \mathrm{H}_{3}, \mathrm{H}_{4}, \mathrm{H}_{6 \mathrm{~b}}\right.$ or $\mathrm{H}_{1 \mathrm{~b}}, \mathrm{H}_{\mathrm{I}^{\prime} \mathrm{b}}$ and $\mathrm{H}_{4^{\prime} \mathrm{b}}$ ); 3.72 (dd, $1 \mathrm{H}, \mathrm{H}_{\mathrm{la}}$ or $\mathrm{H}_{6 \mathrm{a}}, J=7.6,14.9 \mathrm{~Hz}$ ); 3.65-3.61 $\left(\mathrm{m}, 1 \mathrm{H}, \mathrm{H}_{1 \mathrm{~b}}\right.$ or $\left.\mathrm{H}_{6 \mathrm{~b}}\right) .{ }^{13} \mathrm{C}$ NMR $\left(\mathrm{D}_{2} \mathrm{O}\right) \delta 79.8\left(\mathrm{C}_{3^{\prime}}\right) ; 74.3\left(\mathrm{C}_{4}\right.$ or $\left.\mathrm{C}_{3}\right) ; 74.1\left(\mathrm{C}_{4}\right.$ or $\left.\mathrm{C}_{3}\right) ; 69.9\left(\mathrm{C}_{2}\right.$ or $\left.\mathrm{C}_{5}\right) ; 66.8\left(\mathrm{C}_{2^{\prime}}\right) ; 65.4\left(\mathrm{C}_{5}\right.$ or $\left.\mathrm{C}_{2}\right) ; 59.5\left(\mathrm{C}_{4^{\prime}}\right) ; 47.5\left(\mathrm{C}_{6}\right.$ or $\left.\mathrm{C}_{1}\right) ; 43.1\left(\mathrm{C}_{1}\right) ; 39.4\left(\mathrm{C}_{1}\right.$ or $\left.\mathrm{C}_{6}\right)$. HRMS (ESI+) calcd for $[\mathrm{M}+\mathrm{Na}]^{+} 387.0396$. Found 387.0391 .

\subsection{Inhibition studies}

$\alpha$-glucosidase from rice, $\alpha$-glucosidase from baker's yeast, $\beta$-glucosidase from almond, $\alpha$-galactosidase from green coffee beans, $\beta$-galactosidase from Aspergillus oryzae, $\alpha$-mannosidase from jack beans and all substrates (4- or 2-nitrophenyl $\alpha$ or $\beta$-glycopyranosides) were purchased from Sigma. Assays were run at $25^{\circ} \mathrm{C}$ in a phosphate buffer $(25 \mathrm{mM})$ at $\mathrm{pH} 6.8$ using the corresponding 4-nitrophenylglycoside in a total volume of $1 \mathrm{~mL}$. The potential inhibitors were tested at a final concentration of $1 \mathrm{mM}$ and the amount of enzyme of each assay was adjusted so that the system would give the initial rate. After two incubation times ( 5 and $30 \mathrm{~min}$ ) of the enzyme in the presence of the tested molecule, the substrate was added and the optical absorbance was followed at $400 \mathrm{~nm}$. The initial rate was determined, compared with that obtained without the test compound, and the percentage inhibition was calculated. When the percentage inhibition was higher than $33 \%$, the $K_{\mathrm{i}}$ was determined by the Hanes-Woolf method. Four substrate concentrations ( 0.04 to $2.5 \mathrm{mM})$ and four inhibitor concentrations $(0.005$ to $0.8 \mathrm{mM})$ were chosen. The $K_{\mathrm{i}}$ was then calculated from the Michaelis-Menten $\left(K_{\mathrm{M}}\right.$ and four $K^{\prime}{ }_{\mathrm{M}}$ ) constants obtained in the presence or absence of inhibitor. When the percentage of inhibition was between 33 and $10 \%$, the $K_{\mathrm{i}}$ was determined with the equation $K_{\mathrm{i}}=$ $[\mathrm{I}] /\left(K_{\mathrm{M}}^{\prime} / K_{\mathrm{M}}-1\right)$, using only one $K_{\mathrm{M}}^{\prime}$ value.

\section{References and notes}

1. (a) Yoshikawa, M.; Murakami, T.; Shimida, H.; Matsuda, H.; Yamahara, J.; Tanabe, G.; Muraoka, O. Tetrahedron Lett. 1997, 38, 8367. (b) Yoshikawa, M.; Morikawa, T.; Matsuda, H.; Tanabe, G.; Muraoka, O. Bioorg. Med. Chem. 2002, 10, 1547. (c) Yoshikawa, M.; Murakami, T.; Yashiro, K.; Matsuda, H. Chem. Pharm. Bull. 1998, 46, 1339.

2. Lillelund, V. H.; Jensen, H. H.; Liang, X.; Bols, M. Chem. Rev. $2002,102,515$.

3. Szczepina, M. G.; Johnston, B. D.; Yuan, Y.; Svensson, B.; Pinto, B. M. J. Am. Chem. Soc. 2004, 126, 12458.

4. Yuasa, H.; Takada, J.; Hashimoto, H. Tetrahedron Lett. 2000, 41,6615 .

5. Ghavami, A.; Johnston, B. D.; Pinto, B. M. J. Org. Chem. 2001, 66, 2312.

6. Ghavami, A.; Johnston, B. D.; Maddes, M. D.; Chinapoo, S. M.; Jensen, M. T.; Svensson, B.; Pinto, B. M. Can. J. Chem. $2002,80,937$.

7. Ghavami, A.; Sadalapure, K. S.; Johnston, B. D.; Lobera, M.; Snider, B. B.; Pinto, B. M. Synlett 2003, 1259.

8. Ghavami, A.; Johnston, B. D.; Jensen, M. T.; Svensson, B.; Pinto, B. M. J. Am. Chem. Soc. 2001, 123, 6268.

9. Muraoka, O.; Ying, S.; Yoshikai, K.; Matsuura, Y.; Yamada, E.; Minematsu, T.; Tanabe, G.; Matsuda, H.; Yoshikawa, M. Chem. Pharm. Bull. 2001, 49, 1503.

10. Johnston, B. D.; Ghavami, A.; Jensen, M. T.; Svensson, B.; Pinto, B. M. J. Am. Chem. Soc. 2002, 124, 8245.

11. Halila, S.; Benazza, M.; Demailly, G. Tetrahedron Lett. 2001, $42,3307$.

12. Barili, P. L.; Berti, G.; Catelani, G.; Cini, C.; D'Andrea, F.; Mastrorilli, E. Carbohydr. Res. 1995, 278, 43. 
13. Pinto, B.; Johnston, B. D.; Ghavami, A. International patent WO 01/49674 A2, 2001.

14. Benazza, M.; Halila, S.; Viot, C.; Danquigny, A.; Pierru, C.; Demailly, G. Tetrahedron 2004, 60, 2889.

15. (a) Glaçon, V.; Benazza, M.; Beaupère, D.; Demailly, G. Tetrahedron Lett. 2000, 41, 5053. (b) Halila, S.; Benazza, M.; Demailly, G. Tetrahedron Lett. 2002, 43, 815 .
16. Lemaire, M.; Veny, N.; Gallienne, E.; Chenevert, R.; Bolte, J. Synlett 2002, 1359.

17. Yuasa, H.; Takada, J.; Hashimoto, H. Bioorg. Med. Chem. Lett. 2001, 11, 1137. 